\title{
Parameter set for computer-assisted texture analysis of fetal brain
}

\author{
Hugues Gentillon ${ }^{1,2 *}$, Ludomir Stefańczyk ${ }^{1}$, Michał Strzelecki² and Maria Respondek-Liberska ${ }^{3}$
}

\begin{abstract}
Background: Magnetic resonance data were collected from a diverse population of gravid women to objectively compare the quality of 1.5-tesla (1.5 T) versus 3-T magnetic resonance imaging of the developing human brain. MaZda and B11 computational-visual cognition tools were used to process 2D images. We proposed a wavelet-based parameter and two novel histogram-based parameters for Fisher texture analysis in three-dimensional space.

Results: Wavenhl, focus index, and dispersion index revealed better quality for $3 T$. Though both 1.5 and $3 \mathrm{~T}$ images were 16-bit DICOM encoded, nearly 16 and 12 usable bits were measured in 3 and $1.5 \mathrm{~T}$ images, respectively. The fourbit padding observed in $1.5 \mathrm{~T} \mathrm{~K}$-space encoding mimics noise by adding illusionistic details, which are not really part of the image. In contrast, zero-bit padding in $3 \mathrm{~T}$ provides space for storing more details and increases the likelihood of noise but as well as edges, which in turn are very crucial for differentiation of closely related anatomical structures.
\end{abstract}

Conclusions: Both encoding modes are possible with both units, but higher $3 \mathrm{~T}$ resolution is the main difference. It contributes to higher perceived and available dynamic range. Apart from surprisingly larger Fisher coefficient, no significant difference was observed when testing was conducted with down-converted 8-bit BMP images.

Keywords: Histogram, Wavelets, Computer-assisted radiology, Hugues Gentillon, Teleradiology, Prenatal development, Fetal brain, Mazda, b11, Medical cybernetics, Artificial intelligence, Computational visual cognition

\section{Background}

Computer is a non-biological copy of the human brain. As its use increases day-by-day in medicine, various transdisciplinary approaches emerge. Among them is texture analysis, the evolving cybernetics of radiology. This experiment is a translational study seeking to objectively compare the quality of 1.5 -tesla $(1.5 \mathrm{~T})$ versus $3-\mathrm{T}$ magnetic resonance imaging (MRI) of the developing human brain (Fig. 1), in order to determine whether the extra administrative cost is worthy for the patient and the healthcare system. First there was a need to develop an objective methodology to collect and process the data and subsequently justify its necessity and dispel distrust of financial burden.

\footnotetext{
*Correspondence: hugues.gentillon@stud.umed.lodz.pl

1 Department of Radiology and Diagnostic Imaging, Barlicki University

Hospital, Medical University of Lodz, Lodz, Poland

Full list of author information is available at the end of the article
}

\section{MRI replacing USG during pregnancy examination}

In terms of safety, ultrasonography (USG) remains the gold standard for prenatal central nervous system (CNS) imaging [1]. It is used for prevention and diagnosis of congenital malformation [2, 3]. Nevertheless, there are cases which benefit from alternative imaging techniques like 1.5 or 3 T MRI [4-9]. Besides spotting maternal abnormalities, supplemental information from magnetic resonance imaging is also crucial for identification of fetal anatomy and pathology [10, 11]. MRI is also being increasingly used as correlative imaging modality in pregnancy-because it uses no ionizing radiation, has no known teratogenic effects, provides excellent soft-tissue contrast, and has multiple planes for reconstruction and large field of view, allowing better depiction of neuroanatomy in fetuses with large or complex anomalies [12-15]. Compared to magnetic resonance imaging, USG is more affordable and widely used in many countries, as a radiologic tool in routine examination of pregnant women, especially in the detection of fetal anomalies, at about 20th week of gestation [2,3]. On the other hand, 


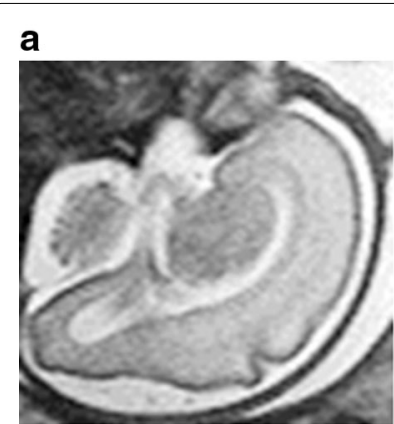

$1.5 \mathrm{~T}$

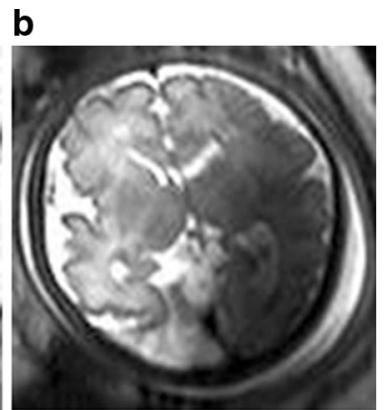

3T
Fig. 1 "Do you see what I see?". Fetal brain. a Left 1.5 T. b Right 3 T. This figure was incorporated and so-titled in this manuscript to illustrate that magnetic resonance (MR) images can be used for evaluation of achromatic vision and sensitivity to change in grayscale quality between different subjects. For fair comparison, this exercise should be blinded. It is recommended to have at least two radiologists, if not available, medically-trained practitioners or any volunteers (blinded) and one examiner (also blinded) to look at the images side-by-side, on two medical-diagnostic monitors, engineered for 16-bit display (same brand/model in natively flat display mode: i.e. without any added enhancements). All in-computer/in-monitor RT (real-time) editing features must be turned off (incl. hardware/software rendering): n.b. 16-bit of true data has more room for "contrast booster" - which is essentially an illusion, as a result of post-editing artifacts, not really part of the image. In this investigation, volunteers were asked to identify the images unlabeled (, of course). It was observed that both images were equally sharp (in pass-through mode), for a normal, naked human eye. There flows the explanation for the research goal to mathematically determine which MR modality actually produces images with more captured details. Both images were 16-bit encoded, and the difference could not be measured via "perceived dynamic range" (visible details). With computer vision software, 1.5/3 T images can be numerically decoded to accurately assess "available dynamic range" (visible/invisible details)

1.5 and $3 \mathrm{~T}$ MRI scanners produce superior CNS images (Fig. 2) but are not cost-effectively built. These units are massive and expensive [16-20]. They are mostly available in big-budget hospitals and wealthy medical research centers. Because of such disadvantages, carrying research with MRI is more likely to be impeded. As a result, little information is known about its relevance to potential beneficiaries. MRI is bureaucratically recommended only if it is essential in some health care systems administrated by a central office (e.g. Sweden) [21]. Physicians are even liable for part of MRI examination cost in some territories (e.g. Poland) $[22,23]$. Thus relevance to beneficiaries is likely to be affected in such health care systems.

\section{What is texture analysis?}

Texture analysis is an artificial process involving quantification of image quality by means of parametric features to characterize regions of interest (ROI) [24-26]. While texture analysis is still far from replacing the clinician eye, some of its features warrant further consideration

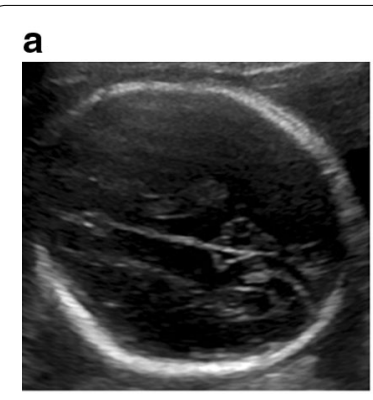

USG

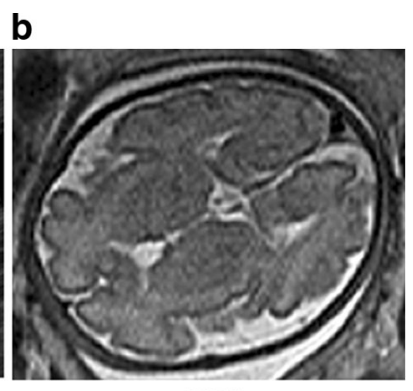

MRI
Fig. 2 Ultrasonography (USG) vs. magnetic resonance imaging (MRI). Fetal MRI was used as a complimentary modality to USG. a USG. b MRI

for integration into medical practice. To this date, texture analysis is used in pre-diagnosis of the globally pandemic disease of tuberculosis [27-29]. In Bangladesh, for example, radiologists and radio-technicians quickly screen patients displaying tuberculosis (TB) signs with CAD4TB texture analysis software [30-33]. This medical application of texture analysis is sponsored by The World Health Organization, at a mere cost of $\$ 3$ per CAD4TB test. Only patients pre-diagnosed TB-positive by CAD4TB software receive the more expensive molecular examination known as GeneXpert TB Diagnosis and Resistance Test [30-33]. In the aforementioned example, an increase in the rate of early- detection of TB has been reported in clinics where texture analysis is used as a pre-diagnostic tool [30-33]. Apart from image encoding statistics, other factors also affect how clinicians perceive medical image appearance: e.g. acutance or subjective quality factor, human eye's contrast sensitivity function, modulation transfer function or spatial frequency response, image displayed height, viewing distance, positioning of the subjects (stand, seat), radiology LCD (liquid crystal display) screen characteristics (anti-glare, glossy, size, resolution, calibration), etc [34-36].

\section{Wavelets-based texture analysis}

Wavelets are texture analysis parameters which classify intrinsic properties of an image into matrix layers, and therefore they are very powerful tools for differentiating and matching patterns in regions of interest. Wavelets are used, for example, in forensic investigation to match fingerprints [37], in diagnostic radiology to detect suspected diseases [38], and in many other applications where digital imaging is needed [37].

\section{Histogram-based texture analysis}

To fully grasp the concept behind the methodology used in this experiment, it is important to first comprehend or at least have a minimum knowledge about the purpose, 
functionality, and usefulness of histogram in digital imaging. Just as the aforementioned brain images in Fig. 1, the quality and texture of every shape in Fig. 3 may not be interpreted in the same way by different pairs of human eyes.

Histogram parameters are digital imaging tools which analyze textural characteristics of a picture by measuring their quality $[39,40]$. Such parameters can differentiate texture by quantifying, for example, resolution, sharpness, noise, actual edge details and artifacts, negative and positive exposures, as well as aliasing and moiréi.e. superimposed patterns in MR images [41-44]; moiré effect is most commonly observed in uncontrolled TV interviews recorded with older cameras-and the interviewees were wearing pinstriped clothing $[45,46]$. In some software, histogram is customized for 8-bit graphical visualization to fit the display of conventional RGB monitors, with luminance value of 0-255 (8 bits $=28=256$ ). In other words, a 16-bit image would be down-sample in order to display its geometric representation on such computational histograms (Figs. 4, 5, 6). A Histogram display in MaZda, on the other hand, does not graphically display pixel values larger than 255 . However, it is able to quantify 16-bit DICOM (Digital Imaging and Communications in Medicine), evaluate quality of different MR modalities, and differentiate ROIs. Is this limit a histogramatic error? No, it is not. A radiology monitor of, probably, the size of a theatre screen is needed to visually display a linear representation of every value in a 16-bit image $\left(2^{16}-1=65,535\right)$. In spite of the graphical limitations, histogram-based texture analysis is more efficient than edge detection for pixel segmentation and identification of region boundaries [47, 48]. Some histogram parameters (e.g. mean, variance, kurtosis, skewness) can be used to differentiate noise and related artifacts from very sharp details such as edges (region boundaries).

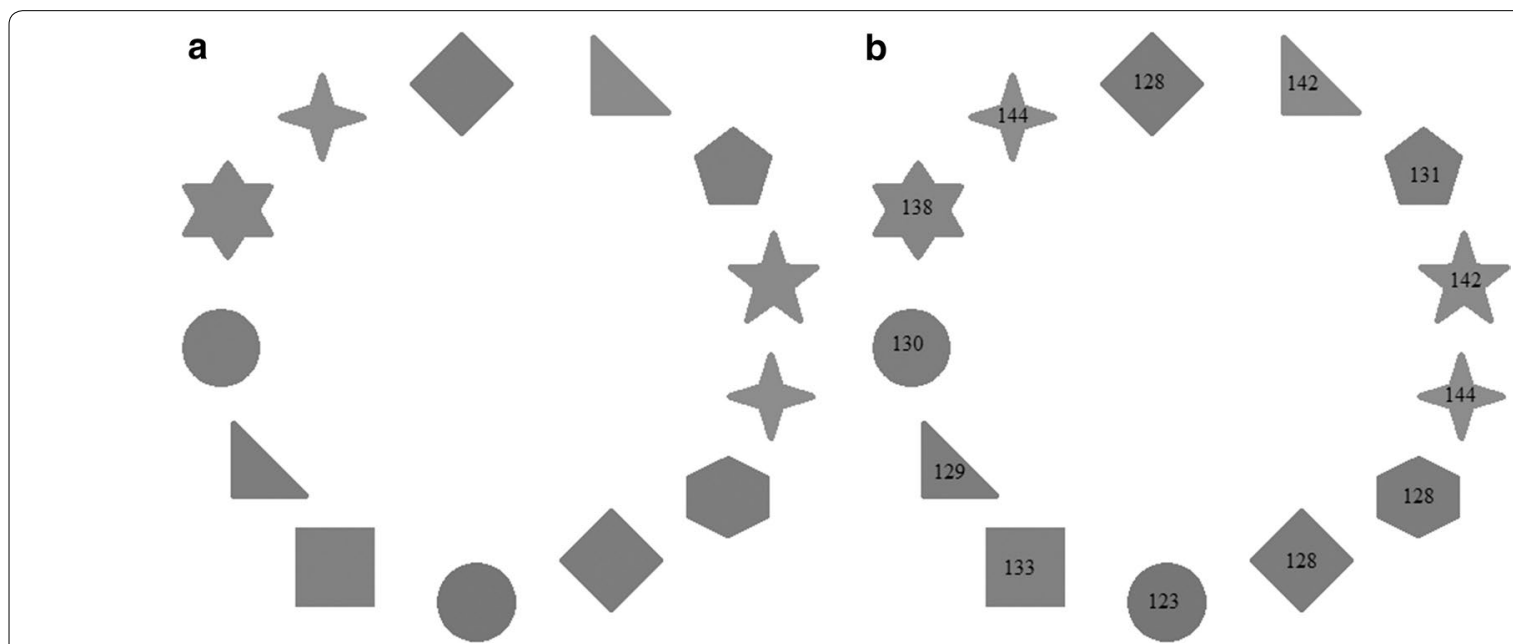

Fig. 3 Varying shades of grey. a Arrange from lighter to darker? b Answers to Puzzle as per measurements from computer vision software. Again, test subjects should be blinded

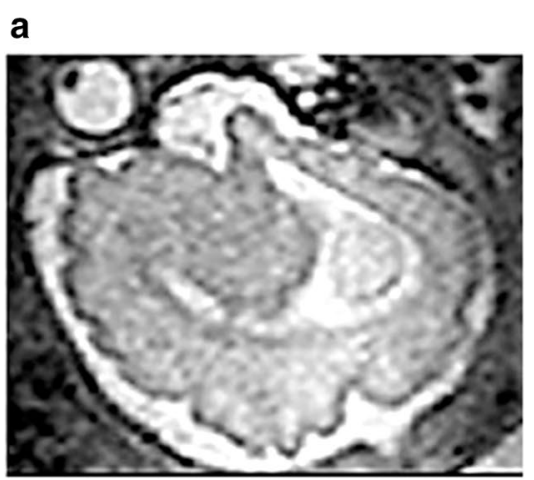

b

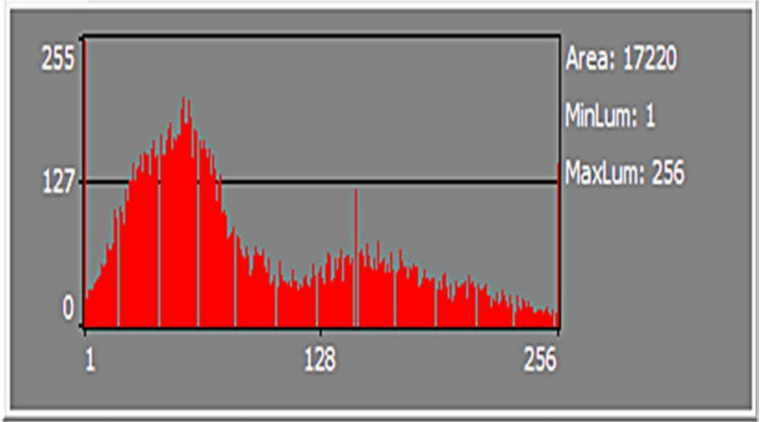

Fig. 4 Histogram representation of fetal brain. a MRI of fetal brain. $\mathbf{b}$ Histogram of the whole image 
a

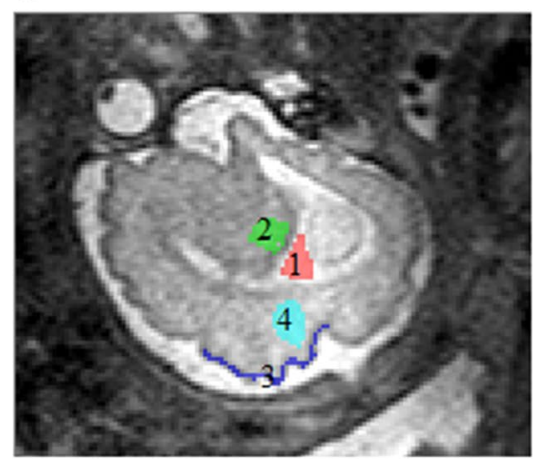

b

\begin{tabular}{lcccc||}
\hline ROI: & 1 & 2 & 3 & 4 \\
Mean: & 248 & 160 & 136 & 214 \\
Variance: & 110 & 179 & 303 & 78 \\
& & & & \\
\hline
\end{tabular}

Mean: average luminance value Variance: contrast measurement

ROl: Regiong of interest; $1=$ ventricles; 2 = thalamus; $3=$ grey matter; $4=$ white matter.

Fig. 5 Regions of interest. a $R O I 1$ ventricles; 2 thalamus; 3 grey matter; 4 white matter. $\mathbf{b}$ Histogram parameters from texture analysis with MaZda

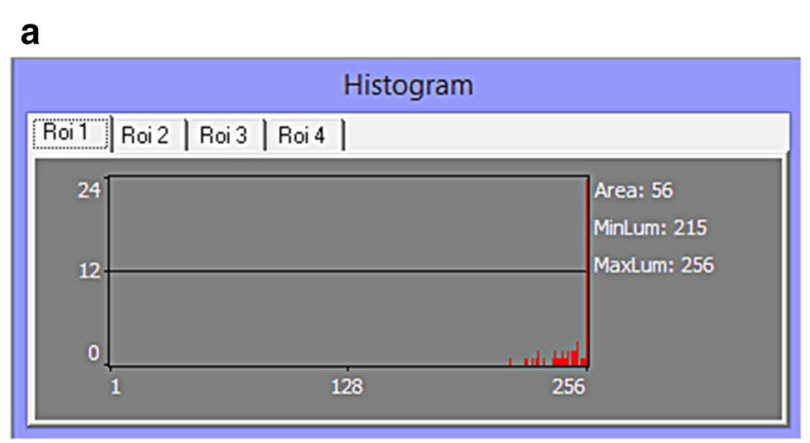

C

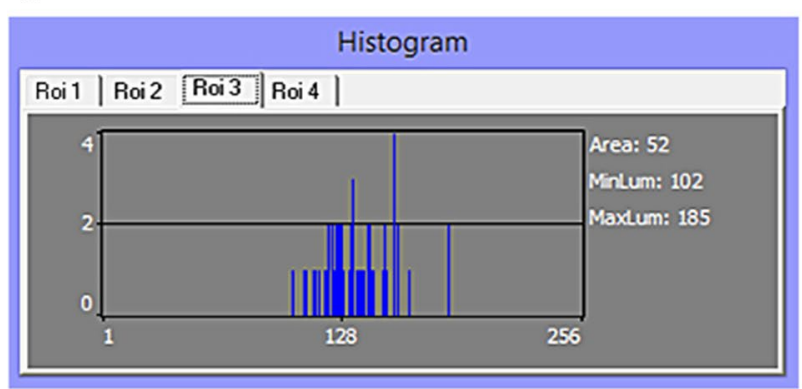

b

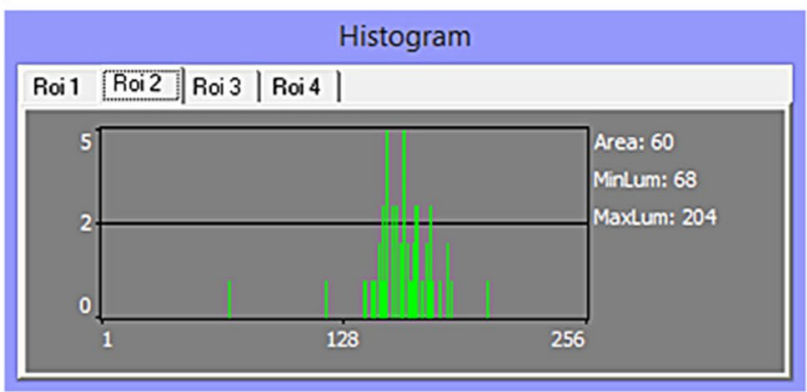

d

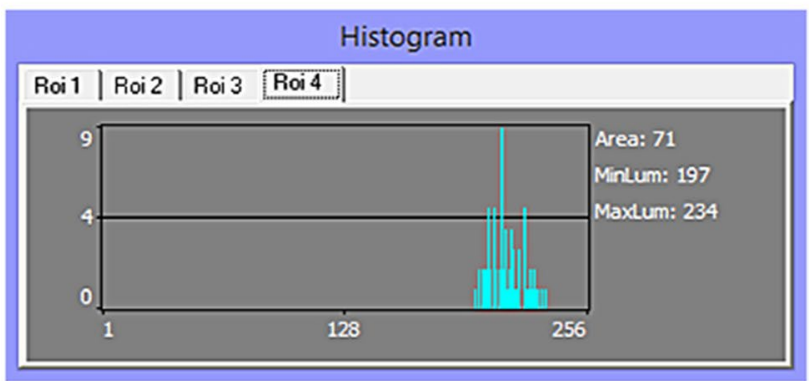

Roi 1: Ventricle; Roi 2: Thalamic nuclei; Roi 3: Grey matter Roi 4: White matter

Fig. 6 ROI analysis. a ROI 1 Ventricles; $b$ ROI 2 Thalamic nuclei; c $R O / 3$ Grey matter; $\mathbf{d} R O / 4$ White matter. There exist several techniques and methods of texture analysis. Non-parametric graphs (e.g. histogram, box plot) would be indeed a simple alternative to conduct this study. As shown in the figures, MaZda does display histogram of 8-bit but not for 16-bit DICOM image. Also, there are issues with drawing conclusions straight from histograms and boxplots. They are pictorial representations and thus are indirect methods. Furthermore non-parametric interpretation may not be as precise and accurate as parametric quantification. Therefore, parametric quantification was used to assess image quality rather than conventional appearance. 3D, non-parametric graphs are also possible with collateral usage of MaZda (version 5) and B11 (version 3.3) in training mode. The problem with training methods is that errors might occur as a result of overtraining the network. Hence, raw analysis was performed

\section{Methods}

This multidisciplinary research was approved by a relevant bioethics committee, consisting of emeritus professors and senior researchers from the Medical University of Lodz (MUL), Barlicki University Hospitals (BUH), Polish Mother's Memorial Hospital Research Institute (ICZMP) et al. Written informed consent was obtained from all subjects, and the methods were carried out in 
accordance with the approved guidelines (see end of the manuscript for registration number). The experiment was divided into three parts.

\section{Research tools}

Firstly, some quality-control checks were performed before designing this research. Images (Fig. 1) were imported and uncompressed in Photoshop CS6 64-bit Extended. The software was set to HDR (high-bit-depth resolution) mode (i.e. floating-point numeric representation: up to 32 bits $\times 3$ channels). RAW-formatted images were simultaneously examined with radiology computer systems (i.e. high-end hardware/accessories, monitors capable to render 16-bit grayscale natively; memoryintensive controller/graphic card, Phillips DICOM Viewer R3.0 SP3, and LCD calibration software). With proper-matching adjustments, difference between the two images were imperceptibly unnoticeable. That was a conundrum which proved the need to carry out qualityassessment research with MaZda and B11.

\section{Trials}

BUH (1.5 T unit) and ICZMP (3 T unit) radiology departments prescribed MRI to patients for further differentialdiagnostic investigation-rather than just a concomitant adjunct to ultrasound. $288 \mathrm{MR}$ images of normal fetal brain were used in the main assessment and $72 \mathrm{MR}$ images of normal fetal brain in the supplementary trials (see Table 1 for further description).

MR images came from MRI studies of different patients who had fetal MRI as a complimentary modality to ultrasonography and echocardiography, to further assess and confirm the diagnosis of suspected anomalies affecting the fetuses as well as the pregnant patients. Most participants were seeking remedies for conditions not related to fetal brain. Research hospitals in Poland are among the forefront pioneers in prenatal diagnosis of congenital malformation, in utero surgery, cardiology and minimally-invasive cardio-surgery [49]. In spite of its stigmatic plight, modern cardiology arguably began in Lodz, Poland, with advances in medical electronics about

Table 1 Sample description

\begin{tabular}{lccc}
\hline & 1.5 T & 3 T & Total \\
\hline A. Main trials & 144 & 144 & 288 \\
B. Supplementary trials & 36 & 36 & 72 \\
Group 1 & 20 & 20 & 40 \\
Group 2 & 16 & 16 & 32
\end{tabular}

A. Main trials 288 MR images, categorized by magnetic field strength of scanner $(144 \times 1.5$ and $144 \times 3 \mathrm{~T})$. B. Supplementary trials $72 \mathrm{MR}$ images $(36 \times 1.5$ and $36 \times 3 \mathrm{~T}$ ). Samples used in the supplementary trials were further divided into two categories, by gestational age: group $120-28$ weeks; group $229-40$ weeks
70 years ago [50]. This background history explained why patients travelled there to seek second opinion rather than just for the known association of cost-effective treatment in Poland. The selected samples were diversei.e. subjects were not closely related (not monozygotic twins, not dizygotic twins, not consanguineous twins, etc). Gestational ages were between 20 and 40 weeks. Patients underwent either $1.5 \mathrm{~T}$ scan at $\mathrm{BUH}$ or $3 \mathrm{~T}$ scan at ICZMP_but not both. It was deemed irrelevant in terms of assessment of image quality as well as for good patient care and fetal rights (i.e. moral and legal rights of human fetuses) [51]. In recent years, physical appearances of subjects (such as eye shapes) have been documented to cause some photography cameras to capture deceptive images in auto-mode-a dysfunction due to faulty-engineering and bug compatibility [52-54]. To our knowledge, such phenomena have not been reported in radiological imaging. Furthermore it is the responsibility of manufacturers to ensure that MR units are capable to capture good quality sequences, regardless of mother and/or fetus phenotypes. In this investigation, the artifacts observed in excluded samples were consequences of fetal movement and inadequate settings by radiographers, not due to phenotypic characteristics. Beside consent-and-publication agreement, the objective of this research did not rationally necessitate broadcasting further descriptions about patients' background/identities (incl. phenotypes and anamneses) [51]. Therefore, such details were omitted in this manuscript as well as in the datasets [51].

MaZda software package 5 (B11 included) was used for quantification of MR images. A wavelet-based parameter (wavenhl) was combined with two novel histogram-based parameters (focus index, dispersion index) to perform Fisher-texture analysis in three-dimensional space. It was hypothesized that Wavenhl could fingerprint (match) regions of interest-i.e. as per human anatomy, the building blocks of normal thalamus, for example, is the same regardless of subjects and MR modalities. Two parameters which were not sensitive to minute variations in phenotypes were favored in this research. Hence, focus index and dispersion index were utilized for assessing image quality in terms of resolution, sharpness, aliasing and moiré, noise, actual edge details and artifacts. MicroDicom was used to extract images from teleradiology network systems and storage media containing raw data of 3 and 1.5 T MRI studies. One DICOM and one BMP (also known as Bitmap: Microsoft Windows DeviceIndependent Bitmap file) were generated from each MR sequence to create four batches of images: $3 \mathrm{~T}$ DICOM, 1.5 T DICOM, 3 T BMP, and 1.5 T BMP. All files were extracted in their native size and resolution. Both 3 and $1.5 \mathrm{~T}$ sequences were processed as Little-Endian, 
Implicit and 16-bit uncompressed DICOM (RAW). All BMP images were encoded with no down- sampling of resolution. Both 3/1.5 T sequences were simultaneously exported as 16-bit DICOM, down-converted to 8-bit BMP (color space: RGB-24: 8 bits $\times 3$ channels), and batch-processed. Note that MicroDicom applied equal, least-significant-bit (LSB) degradation to both $3 / 1.5 \mathrm{~T}$ BMP images. The quality and the information loss in both 3/1.5 $\mathrm{T}$ images were assessed with MaZda, a texture analysis software which measures image statistics in various file formats $[4,5,55]$. Histogram is the only feature in MaZda version 5, to numerically measure available dynamic range in 16-bit images. Histogram parameters were then used to calculate focus index values (skewness-to-mean kurtosis ratio) and dispersion index values (variance-to-mean ratio), which in turn serve as tools to evaluate the retention of stored, tonal details in BMP ROIs-compared to the same ROIs in the original DICOM file (Figs. 4, 5, 6, 7, 8; Additional file 1: Dataset 1). MaZda measured nearly 16 and 12 usable bits in 3 and 1.5-T DICOM images, respectively (Fig. 8; Additional file 1: Dataset 1). No significant difference was observed when testing was conducted with degraded BMP images (max. 8 bits in both) (Fig. 8; Additional file 1: Dataset 1).

Usable bit (stored bit) was used as a parametric factor rather than allocated or high bit, as the primary research

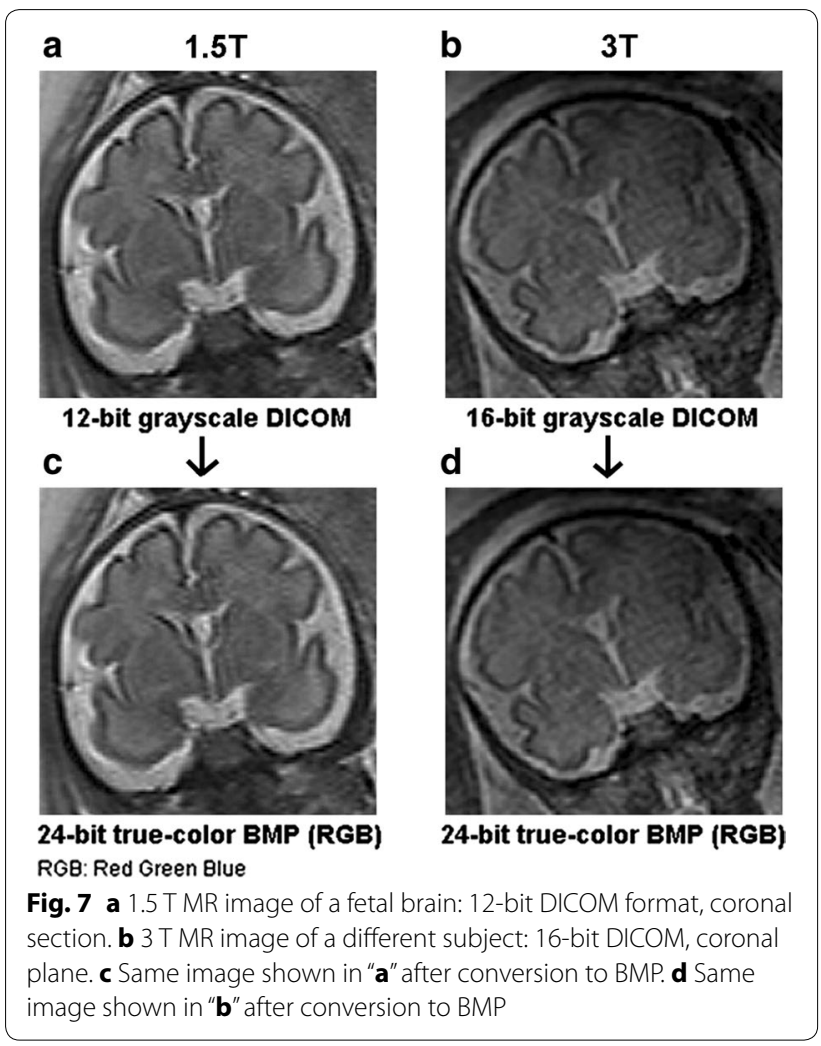

interest was a quest to assess quality in terms of actual, captured details-free from illusionistic embellishments. In other words, a DICOM file may appear as or show properties display of a 16-bit image on a computer; when in reality it only holds 12 bits of the real thing. Many of the measured 16-bit DICOM images contain much less than 65,536 tonal patterns per pixel. It is due to the fact that not all allocated bits contain real captured details but bogus data. Reported usable bit can be simply obtained with any software which has a DICOM header viewer or editor. In this experiment, reported usable bits (in the DICOM headers) were first checked with MicroDicom. Then these metadata were double-checked and subsequently measured with MaZda to determine actual stored bit in every image. Measured usable bit does not always correspond to reported usable bit. Such discrepancies resulted from rounding errors in MR units. Thus the choice to go with measured usable bit was factually validated (Fig. 8; Additional file 1: Dataset 1). Images with $4-5 \mathrm{~mm}$ thickness and highest measured usable bits were selected. For texture segmentation, coronal plane was ideal because more images could be obtained from sequences with all four visible ROIs (i.e. thalamus, ventricles, grey matter and white matter). Furthermore it is worth mentioning that histogram and wavelets measurements depend on MRI signal types: e.g. "spin-lattice" relaxation time (T1 or T1 weighted image); "spin-spin" relaxation time (T2 or T2 weighted image); proton density (PD) (see Additional file 2: Dataset 2, Additional file 3: Dataset 3 for additional details).

\section{Index of focus}

Focus index or index of focus (abbreviated: I-focus) is neither an integral parameter in MaZda nor in B11. It has been scarcely used before to measure perceived quality and realness of wood images [56], an embellishing technique for enhancing appearance of floor murals, backdrops and wall decorations used in low-cost construction. Focus index is calculated by dividing skewness by kurtosis. Skewness is a parameter which measures surface symmetry or lack of symmetry, imperfections, scanner misalignment. It indicates the even portion of a surface and the direction of distortion in the uneven portions (negatively and positively skewed). On the other hand, kurtosis is a measurement of the peakedness and flatness of fine details and edges where textural variations occur. When an image is in focus, edge elements or objects are sharp. Focus index combines these two parameters to thus assess quality by measuring focal or perspective distortion and sharpness. An image may be sharp but distorted and vice versa. In magnetic resonance imaging, focus index varies from one perspective plane to another (i.e. axial, coronal, sagittal). Ideal focus index is zero or close to zero $[56,57]$. Sharpness quality for focus 


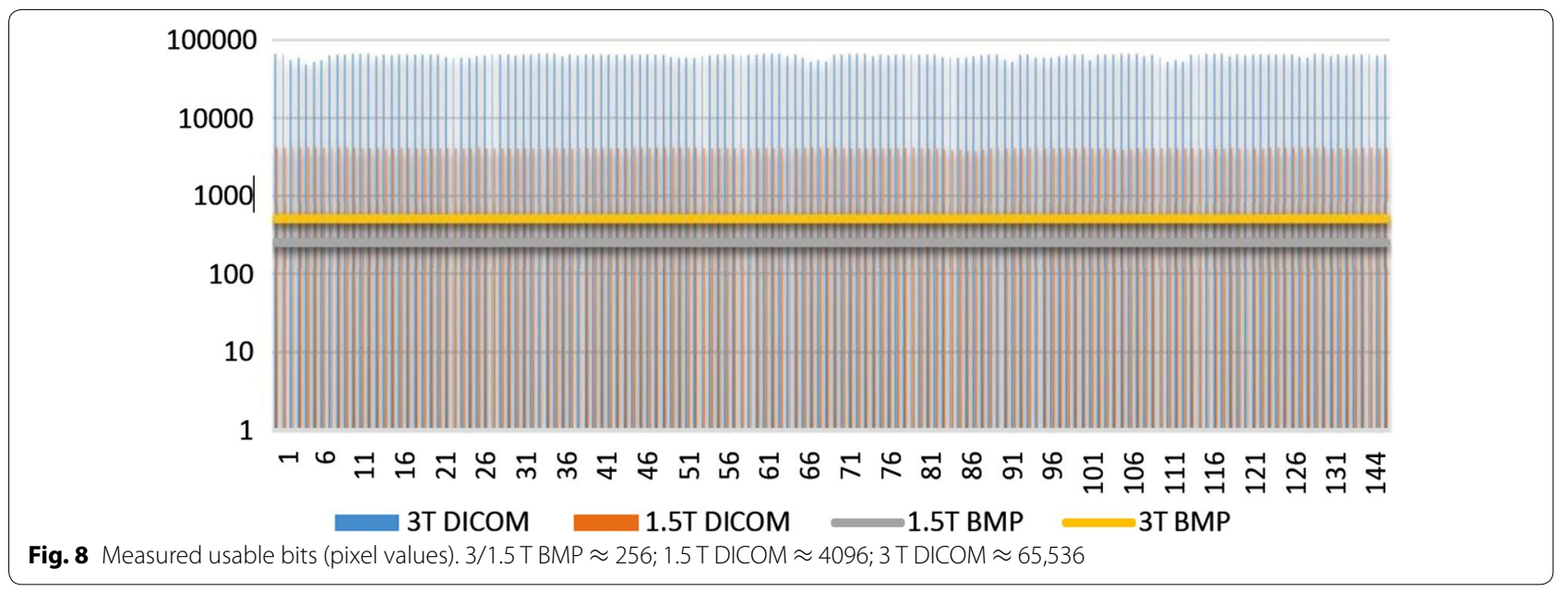

index values outside the range of $[-1,+1]$ can be easily perceived by the naked, normal human eye. It is also important to note that focus index alone does not determine image quality. The common pitfall of the skewness and kurtosis is their sensitivity to artifacts like noise, grain, post-editing sharpening and Gaussian blur $[56,57]$.

\section{Index of dispersion}

Index of dispersion [also known as variance-to-mean ratio (VMR)] is a measurement used to determine the clustering or dispersion of a luminance. In this research, VMR was utilized to quantify the volatility (difference) of each individual ROI (ventricle, thalamus, grey matter, white matter). The larger the difference between the coefficients of dispersion (dispersion index values) the greater the variability between the ROIs. Variance is the VMR component which measures contrast and boundaries (edges) in a surface. Like kurtosis and skewness, variance is also affected by noise [58]. Variance is the difference in luminance and/or color that makes an object or its representation in an image or a ROI to display distinguishable. In other words, variance is a histogram parameter which can be used to determine contrast volatility in an image. Finally, mean is a reflection of the average grayscale luminance in MR images. It is directly proportional to the stored bits of an image.

\section{Resolution}

$3 \mathrm{~T}$ scanner is by default constructed to capture more details than $1.5 \mathrm{~T}$. Its higher magnetic strength, when used properly, can also overcome problems with signalto-noise ratio (SNR). Therefore, $3 \mathrm{~T}$ units can conditionally produce image with more information and less distortion. This superiority is technically due to denser sampling of K-space for the same size of field of view (FOV), resulting in increased resolution. Nonetheless, several other factors affect the visual interpretation of the MR image which reaches the radiologist via medicaldiagnostic LCD (e.g. acutance, physical and technical unit settings, SNR, FOV, extended knowledge of elements affecting perceived appearance, realness, sharpness, etc) [59]. "Sharper" does not always signify "more details" and "more details" does not always signify "sharper." Quality depends on more factors than just "more details". A MR image can have "higher resolution" and "more details" and be not sharp or be degraded by artifacts and thus of poor quality. Unless its quality can be restored with computer vision and/or editing tools, such an image would be useless for live medical application. In clinical practice, MR units have been used to detect anatomical, functional and molecular anomalies. MR imaging modalities and the quality of their products are crucial because physicians not only rely on them to reach a firmly conclusive diagnosis but also to make final interpretation to administer therapeutic care; which if wrong can lead to malpractice litigation.

\section{Fisher coefficient}

One of the most promising application of texture analysis in medicine is early detection of tumorous signs and prophylaxis of cancer. A methodology of interest to this research was Fisher texture analysis. It has been used before in medical research to discriminate healthy from benign or malignant tissue. In this experiment, it was necessary to develop a modified calculation of Fisher coefficient-coined as "feature-selection coefficient" (Eq. 1). It was derived from an amalgamation of Fisher coefficient and statistical principles of analysis of variance (ANOVA).

$$
F=\frac{D^{2}}{V^{2}}=\frac{\frac{1}{1-\sum_{k=1}^{K} P_{k}^{2}} \sum_{k=1}^{K} \sum_{j=1}^{K} P_{k} P_{j}\left|\mu_{k}-\mu_{j}\right|^{2}}{\sum_{k=1}^{K} P_{k} V_{k}^{2}}
$$


In Eq. 1, feature-selection coefficient is derived from Fisher coefficient and ANOVA, where D is between-class variance, $V$ within-class variance, $P_{k}$ probability of feature $k, V_{k}$ variance value of feature $k$ in given class and $\mu_{k}$ mean value of feature $k$ in given class (see MaZda user manual, http://www.eletel.p.lodz.pl/mazda/download/ mazda_manual.pdf for further details).

Resolution already indicated that $3 \mathrm{~T}$ captured more details than $1.5 \mathrm{~T}$. However, it was still crucial to test the differential capability of each modality in their own defined three-dimensional (3D) space (with dispersion index, focus index, wavenhl) and the effects of image compression on individual parameters. Features extracted from MaZda histogram were plotted on B11 $\mathrm{XYZ}$ space along with two controls [3T: $\mathrm{C}_{\min }(0,-1$, $0), C_{\max }(5500,1,120,000) ; 1.5 \mathrm{~T}: \mathrm{C}_{\min }(0,-1,0), \mathrm{C}_{\max }$ $(307,1,120,000)]$. Note that only the $\mathrm{x}$-axis is different. This comparative method ensured that 1.5 and $3 \mathrm{~T}$ are fairly measured within the resolution limit of the tested samples. The B11 program computed $\mathrm{F}$ from ANOVA derived parameters $(\mathrm{F}=\mathrm{D} 2 / \mathrm{V} 2$, where $\mathrm{D}=$ betweenclass variance and $\mathrm{V}=$ within-class variance). Then it placed ventricles, thalamus, grey matter, and white matter within a 3D space. By themselves, $C_{\min }$ and $C_{\max }$ yielded a maximum $\mathrm{F}$ of $10 \mathrm{E}+6$ and a misclassified data error (MDE) of $0 \%$ for both 1.5 and $3 \mathrm{~T}$. The effect of sample-mismatching on $\mathrm{F}$ and the software mechanics were closely studied with control parameters and 3D graphs. It was observed that changing one $\mathrm{C}_{\max }$ to $[0,-1$, $60,000]$ while others remain constant caused a $25 \% \mathrm{MDE}$ and $\mathrm{F}$ value dropped to 18 .

\section{Results}

Wavenhl, focus index (skewness-to-kurtosis ratio) and dispersion index (variance-to-mean ratio) reveal better quality for $3 \mathrm{~T}$ (Fig. 9). Though both 1.5-T and 3-T images were 16-bit DICOM encoded, nearly 16 and 12 usable bits were measured in 3-T and 1.5-T images, respectively. Four bits in all $1.5 \mathrm{~T}$ images were padded. Such K-space encoding methods appeared to reduce noise, by adding illusionistic details which are not really part of the image. In contrast, all $3 \mathrm{~T}$ images were zero-bit padded. This encoding technique provides space for storing more details and increases the likelihood of noise but as well as edges-which in turn are very crucial for differentiation of closely related anatomical structures. Both encoding modes are possible with both units, but higher $3 \mathrm{~T}$ resolution is the main difference. Apart from surprisingly larger Fisher coefficient (Fig. 9), no significant parametric difference was observed ( $p>0.05$ ) when DICOM files with 12 and 16 stored bits were degraded to 8-bit BMP (Additional file 2: Dataset 2). MaZda measured an equal detrimental loss of quality in both 1.5-T and 3-T BMP images (Additional file 1: Dataset 1); but ROI discrimination was still better in $3 \mathrm{~T}$. In both pre- and post- compression, $\mathrm{F}$ was larger for $3 \mathrm{~T}$. Unexpectedly, F was even better in the degraded images.

\section{Supplemental trial runs}

A preliminary trial was carried out as per recommendations in MaZda and B11 user manuals/tutorial guides (Fisher coefficient computation). The manufacturer's recommended method was tested with two different frames (images), extracted from a single $3 \mathrm{~T}$ sequence and two different frames from a single $1.5 \mathrm{~T}$ sequence. Then two overlapping ROIs were selected and grouped-per anatomical structures in each image (i.e. 2 ROIs of thalamus, 2 ROIs of ventricles, and so on). The outcome is delineated later in this section herein. It is also important to note that some aspects of this research were out of the researchers' control-meaning that radio-technicians performed MRI examinations that attending physicians and hospitals deemed necessary and safe for their patients. Thus the images collected were limited to the setting modes delineated in the MRI prescriptions and hospital-approved imaging protocols-which in turn is contingent upon routine checkups, suspected pathologies and gestational age-as well as regional-clinical practice. The latter varies from one location to another. In the USA, for example, Food and Drug Administration (FDA) does not approve MRI with intravenous gadolinium-based contrast agents for use during pregnancyalthough nobody has yet discovered any hazards to fetus. A slice thickness of $3 \mathrm{~mm}$ is commonly used for routine fetal brain examinations, while 4-mm is used for other organs. In all collected samples, magnetic resonance imaging was performed as an adjunct to ultrasound for clarification of conditions mostly not related to brain anomalies. Throughout this research, the selection consisted of 1.5 and $3 \mathrm{~T}$ sequences which were within the acceptable specifications: no apparent brain defects; slice thickness: 3-5 mm; repetition time: $800-2100$; echo time: 1-95; imaging frequency: 60-130; pixel bandwidth: 400-1200. Final sorting step necessitates pixel-by-pixel processing of 16-bit grayscale and then identification of parameters with lowest standard deviation. What does this sorting algorithm entails. It means that all MaZda parameters limited to 8-bit and 12-bit feature extraction were excluded. From the parameter list matching the criteria, three (skewness, kurtosis, and mean) were chosen for the B11 tests because the measurements were fixed every time the process was repeated and had lowest standard deviations between frames. Furthermore skewness, kurtosis, and mean parameters had measurements that were very close to the average values. These extracted features were then analyzed in 3D space with 

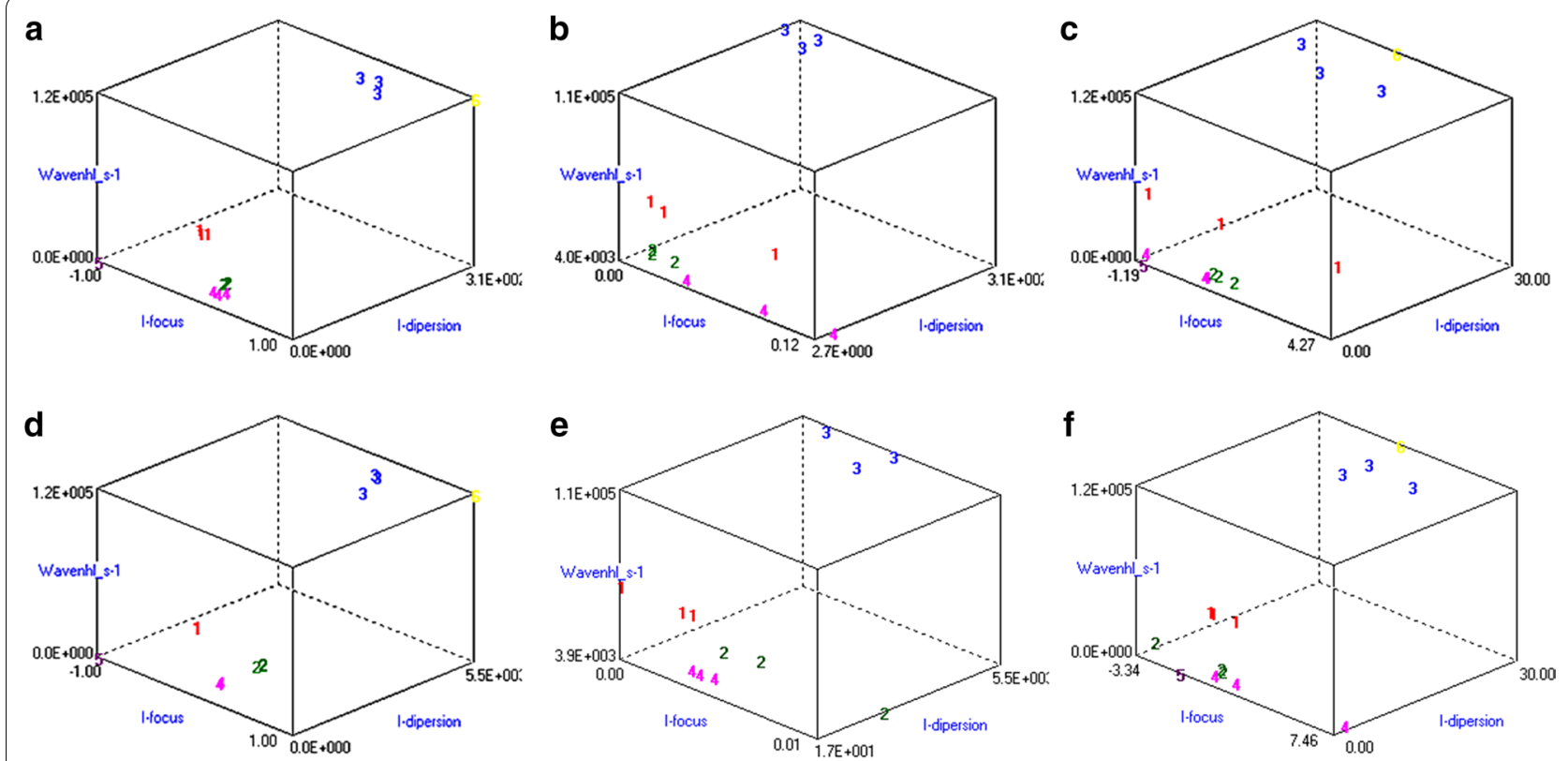

1(red): ventricle; 2(green): thalamus; 3(blue): grey matter; 4(pink): white matter; 5(purple): Control minimum (Cmin); 6(yellow): Control maximum (Cmax)

Fig. 9 Graph showing difference between ROls. Raw-data analysis was performed to compute F with 1-nearest-neighbor (NN) classification and no feature standardization. Same samples and ROIs were used in both pre- and post-image compression. a $1.5 \mathrm{~T}$ uncompressed DICOM; Fisher coefficient computation with controls: $\mathrm{Cmin}=(0,-1,0) ; \mathrm{Cmax}=(307,1,120,000) ; \mathrm{F}=426.0 ;$ MDE $=0 \%$. b Same 1.5 T samples: $z 00 \mathrm{med}$ in, mostly on the $y$-axis. c same 1.5 T after compression to 8 bits; Fisher coefficient computation with controls: $\mathrm{Cmin}=(0,-1,0) \mathrm{Cmax}=(30,1,120,000)$; $\mathrm{F}=776.0 ; \mathrm{MDE}=5.56 \%$. d 3 T uncompressed DICOM: F = 1787.0; MDE =0\%. e Same 1.5 T samples: zoomed in, mostly on the $y$-axis. f Same $3 \mathrm{~T}$ after compression to 8 bits. $3 \mathrm{~T}: \mathrm{F}=2344.3 ; \mathrm{MDE}=0 \%$

B11. For images extracted from the same 1.5 and $3 \mathrm{~T}$ sequences, RAW analysis yielded a maximum Fisher coefficient of $\mathrm{E}+5$ and perfect $0 \%$ misclassification error for both imaging modalities. B11 plugin was used to generate and examine the result on a graph (Fig. 10). Though the graph appears as texture analysis of a single image, each number is actually from two different frames overlapping in three-dimensional space. The software guidelines as well as its engineers were consulted regarding B11 limitation. It turned out that B11's Fisher Coefficient (F) is internally computed from modified ANOVA statistics-by customizedly dividing a nominator (difference between regions) over a denominator (difference within regions). We did not investigate further and neither modify the computation mechanics of Fisher Coefficient, as the software license did not authorize us to do so. Larger F was construed to mean that there is more difference between ROIs. For the purpose of this research, straight-forward comparative test with Fisher coefficient method was inconclusive, as F was the same for comparison between 3 and 1.5 T ROIs (see Additional file 3: Dataset 3-appendix 1, appendix 2, appendix 3 for further details).

The software maker recommended to use different frames from different MR sequences and/or studies.
Different sequences from different patients were scored (Fig. 11). Fisher coefficient was 897.4 for $3 \mathrm{~T}$ and 144.1 for $1.5 \mathrm{~T}$. It is important to note that proton density (PD) sequence is very different from T2. The trial was re-run with two 1.5 T T2 HASTE (half-Fourier acquisition single-shot turbo spin-echo) sequences from two different patients; and F was 448.6. The software maker did not have an immediate solution for the B11 limitation and thus recommended to use the software when comparatively testing many images from different sequences.

In technically controlled settings, the software maker suggestions are feasible. However, in medical practice, it is not a viable process. It would be detrimental to get so many MR studies from a single patient for ROI analysis.

\section{Grayscale quantification with histogram-based parameters} There are misconceptions about the usefulness of grayscale quantification and its applications in medicine. While this area of diagnostic radiology is still emerging and under-documented-grayscale texture analysis with histogram-based parameters is already proven to be very useful for differentiation of benign from malignant thyroid cancer, for example. Such parameters can be used to detect micro-calcifications inside nodules-which is an early sign of thyroid malignancies. In this research, 

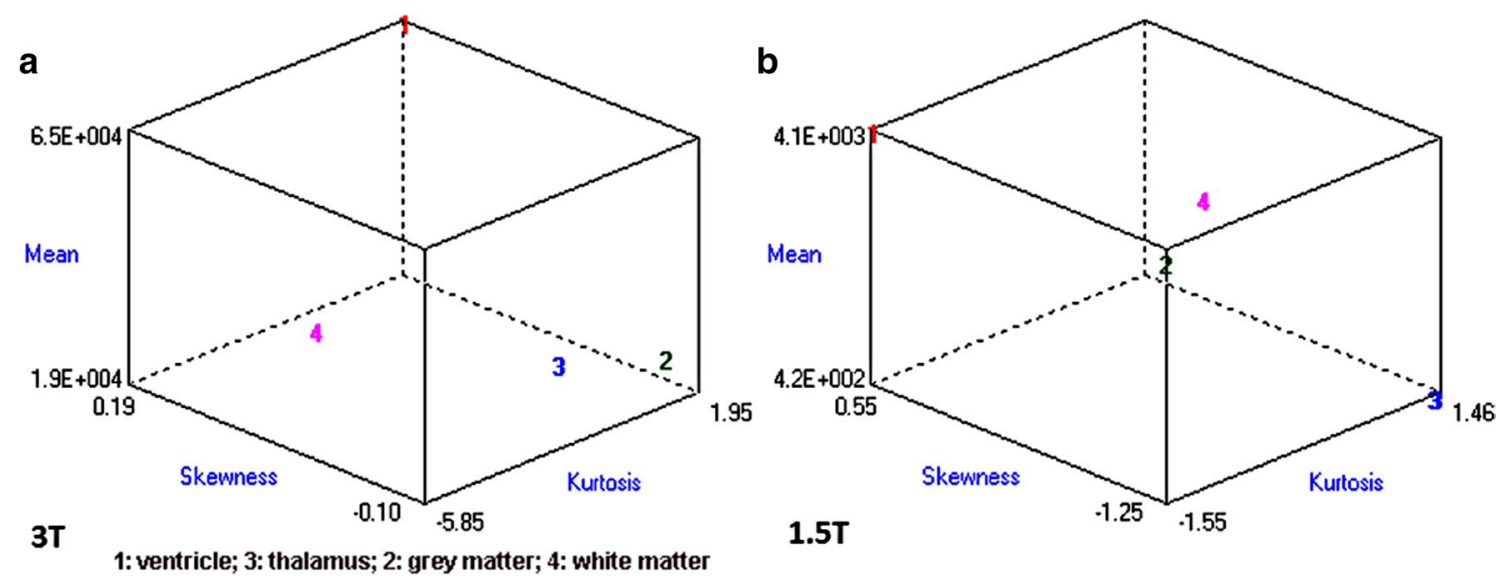

Fig. 10 a Difference between ROls for 3 T with two images from same T2 sequence (same patient). 1 ventricle; 3 thalamus; 2 grey matter; 4 white matter. b Difference between ROls for 1.5 T with two images from same T2 sequence (same patient). 1 ventricle; 3 thalamus; 2 grey matter; 4 white matter
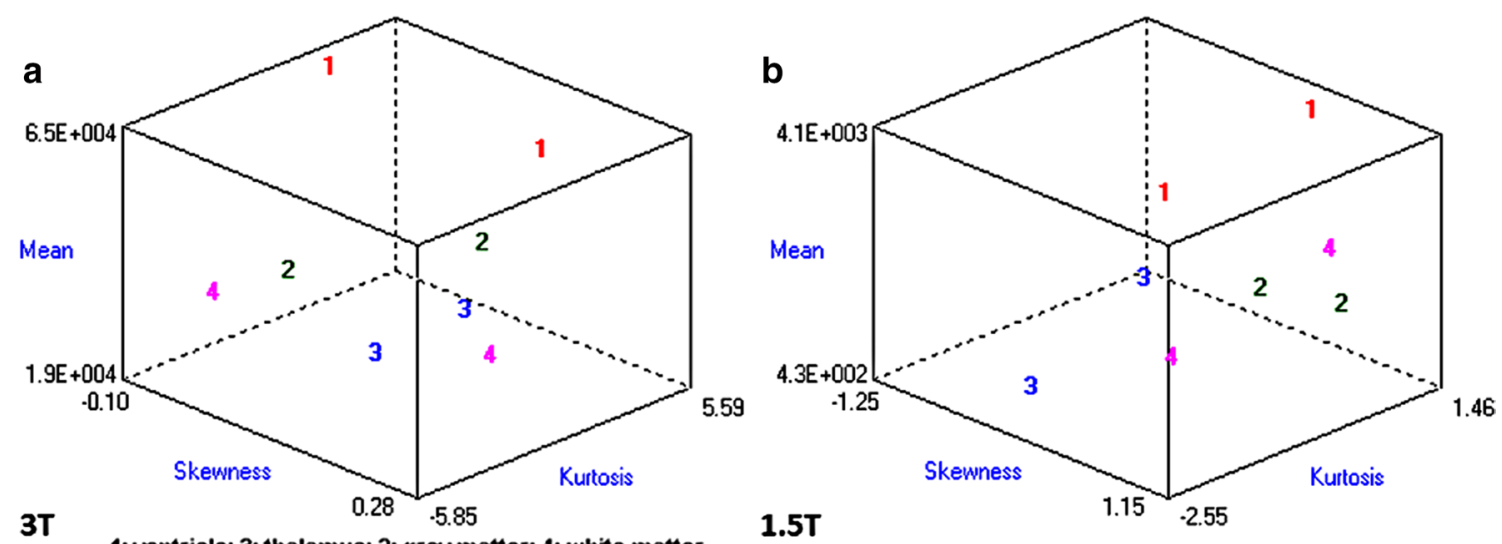

Fig. 11 a Difference between ROls for 3 T with two images from different T2 TSE [Turbo spin echo sequences (different patients)]. 1 ventricle; 3 thalamus; 2 grey matter; 4 white matter. b Difference between ROIs for $1.5 \mathrm{~T}$ with one image from PD and one from T2 HASTE sequence (different patient). 1 ventricle; 3 thalamus; 2 grey matter; 4 white matter

histogram parameters was used to calculate focus index ratios (skewness-to-mean kurtosis ratio) and dispersion index values (variance-to-mean ration). What is the logical meaning of using such parameters in magnetic resonance imaging? Histogram-based parameters don't just generically measure intensity of grayscale and signal-tonoise ratio (SNR). Such parameters can also be used for morphometric measurements, texture segmentation and discrimination. The functionality of focus index can be simply understood from its numerator and denominator. In computational- visual cognition and imaging statistics, skewness is a numerical measurement of symmetry and dissymmetry in an image. Kurtosis, on the other hand, is a histogram parameter that is sensitive to Gaussian blur and out-of-focus blurs (indirect sharpness), noise and grain (simulated sharpness), peakedness and flatness of fine details and edges (due to image resolution), chromatic aberration blur (optical distortion) and other artifacts. Focus index would probably sounds novel to most or perhaps all readers of this article. Yet, it is sparsely used in reality-rendering imaginary to measure realness (naturalness) of surface perception-and thus the phenomenon that human eye does not always sees what it thinks it does. Applications of focus index include photo-realistic rending, optical/visual illusion, methatetic continuum (change in stimulus quality) and indiscernible visual perception between natural materials and replicas (e.g. outdoor backdrops, floors and murals) used in filmmaking and architectural construction. A pitfall with focus index is its susceptibility to digital and mechanical artifacts 
recorded by imaging equipment but not really part of the image. How was the problem solved? Perceived quality of photo-realistic prints was previously documented to be optimally construed as real-when focus index is zero or nearly zero but within -1 and +1 interval. Furthermore focus index and dispersion index were concomitantly used to rule out noise (artifacts) from true signal (actual anatomy). The definition of dispersion index can be technically interpreted in the difference between its dividend and divisor as well as the resulting quotient. Variance is a numerical measurement of contrast and captured details in an image; and thus it is affected by resolution. Mean is the average luminance derived from stored bit value in an image (ROI in this case). Mean may be misunderstood as a mere measurement of pixel values, but herein it also served as a reference value for the variance. Stored bit is not to be confused with allocated bit and high bit. The stored bit value reported in the DICOM tag did not always correspond to that measured with MaZda. A large gap between the variance and the mean could be an indication of fine details or edges (e.g. anatomical boundaries or pattern changes within anatomical structures); or it could also be due to randomly occurring noise and/ or unwanted artifacts. Both 1.5 and $3 \mathrm{~T}$ images were encoded in 16-bit DICOM container. Why don't they have the same quality? Not all bit slots necessarily contain true details (actual anatomy). In our test, converting (uncompressing) 16-bit Lossless JPEG DICOM to 16-bit RAW DICOM did not improve quantification of bit slots which actually contain anatomical details-though the RAW file was larger. If stored bit was initially 12 , it was still 12 after uncompression. MR scanner settings are also known to digitally and mechanically affect quality. Therefore, 3 and $1.5 \mathrm{~T}$ images were closely matched by matrix size, field-of-view (FOV), slice thickness, phase and frequency encodings. Besides magnetic strength and speed, the quality of captured details also depends on the voxel size and thus the resolution of 1.5 and $3 \mathrm{~T}$ (in the collected samples, $256 \times 256$ and $446 \times 446$ respectively). Separating noise and discretization artifacts from true signals is a time-consuming editing process. A more efficient solution was to get rid out of heavily noisy samples. So doing does not necessarily imply that we think that noise is always bad. Despite its degradative nature, noise can also improve visual appearance of an image. Dither, for instance, is in-machine (real-time) or postediting added noise to filter some unwanted artifacts (e.g. off-resonance bandings, posterization)-in order to improve acutance (sharpness) and therefore visual perception. By narrowing the reference range of the focus index quotient to $[-1,+1]$, a large number of noisy samples were excluded, as a result of displaying characteristics of random quantum mottle (grainy appearance).
Hence the larger variance observed in $3 \mathrm{~T}$ parametric measurements are likely due to sharply captured anatomical details with fine edges-rather than digitally and mechanically generated noisy artifacts.

\section{Computation of difference between ROIs}

It would be subjective to just judge the quality by simply comparing 1.5 and $3 \mathrm{~T}$ images on histogram graphs. As a remedy, other comparative tests were developed and carried out. In Fischer coefficient, the numerator (difference between the ROIs) was the quantification wanted. Hence analysis of variance was subsequently performed to quantify the difference between 3 and $1.5 \mathrm{~T}$ ROIs. The parameters were then imported into STATISTICA version 10 for further statistical processing and analysis. All the results were exported to excel, and a dataset was generated (Additional file 4: Dataset 4). $3 \mathrm{~T}$ images $(\sim \mathrm{E}+15)$ had much higher ROI variations than $1.5 \mathrm{~T}$ images $(\sim E+10)$. In terms of parametric quantification, the increased variability between ROIs revealed that $3 \mathrm{~T}$ is a better discriminative tools than $1.5 \mathrm{~T}$ (Fig. 12). Focus index values and dispersion index values were used respectively to measure sharpness distribution and statistical dispersion (Figs. 13, 14, 15, 16). The focus index graph shows that $3 \mathrm{~T}$ images have better sharpness and better focus in both groups. As per dispersion index graph, $3 \mathrm{~T}$ is also better in terms of spatial resolution. In all measurements, the results were statistically significant with $\mathrm{p}<0.01$ (see Table 2).

\section{Discussion}

In this experiment, the findings revealed that 8-bit BMP actually yielded higher $\mathrm{F}$ than the original 16-bit DICOM. Larger F coefficient does not necessarily mean better quality. Nonlinear discriminant analysis (NDA), linear discriminant analysis (LDA), and principal component analysis (PCA) can generate low or large $\mathrm{F}$ when B11 is overtrained [60]. Thus RAW data analysis was performed, as it is more constant than NDA, LDA, and PCA transformations. With featured standardization turned-off, RAW data analysis calculated same F values each time measurements are repeated. In this case, larger F from 8-bit BMP is unlikely to be due to overtraining problem in the network. It is rather due to squeezing tricks employed in lossy compression. While ROIs appear more uniform, some fine details get discarded. In MaZda texture analysis software, histogram can indeed measure regional tonality directly from image statistics rather than from secondary visual means such as graphical appearance on a monitor screen; which is susceptible to eye limitations (e.g. acutance, screen size, etc). Thus 16-bit image can be accurately mapped three-dimensionally from intensity value 


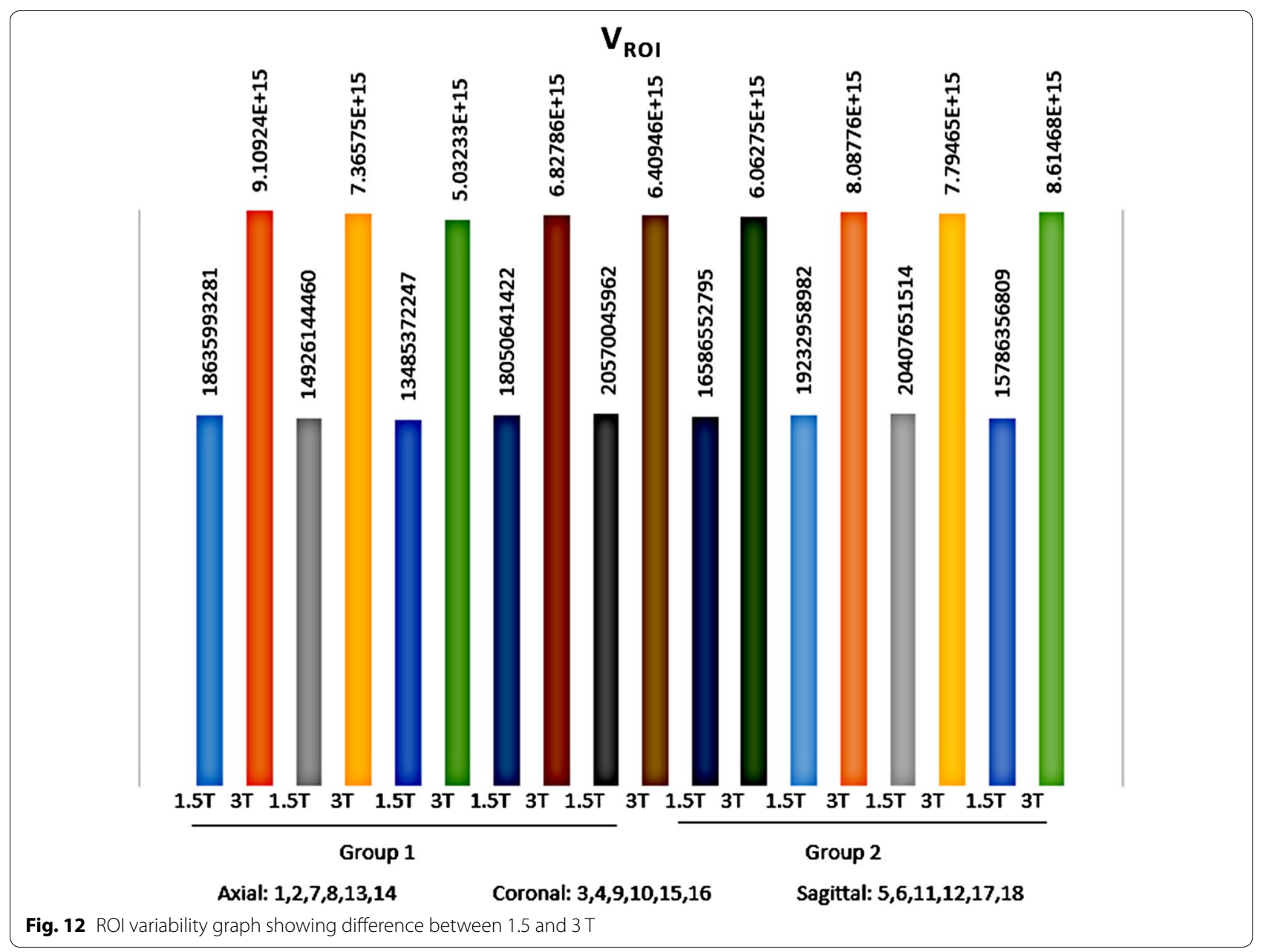

of 0 to a maximum intensity of 65,535 ; and variance provides information about details, sharpness and edges in the image. $3 \mathrm{~T}$ is technically able to capture more details due to higher resolution. Nevertheless, higher resolution does not always guarantee better quality and neither does it always mean more details. Without proper unit settings to booster available dynamic range, resolution is just a mere increment in the cost of storage media. Available dynamic range of an image is the information that is not seen until it is actually used. The original encoding bit space is crucial for storage of captured details. In 8-bit space, for instance, it is mathematically impossible to recover fine highlight details such as blue sky in an indoor image of a blown-out window on a bright sunny day. Lowering exposure shows grey sky. In 12- or higher-bit space, the sky should be still blue. This is where available dynamic range comes in play. Wavelets, on the other hand, were not used for measuring quality but for double-checking and matching regions of interest (i.e. ventricles, thalamus, grey matter, and white matter). Concomitant texture analysis with wavelets and histogram allows accurate classification of closely related anatomical structures in the brain during development, and thus an important set of tools for early detection of tissue changes in the brain. For example, grey matter is vastly present in the brain. It can be difficult for the unaided human eye to trace the boundaries of hypothalamic nuclei on a prenatal magnetic resonance image. It is due to the fact that grey matter is also present deep inside the cerebrum and inside the thalamus, hypothalamus, and the basal ganglia. Even with the fetal brain fully formed, it is still elusive for the human eye to delineate and differentiate these anatomical structures during pregnancy. In the overexposure example, the human eye would see the exact same blown-out region with no obvious quality discrimination in either 8-bit or 16-bit space, while histogram and wavelets would pick up the hidden details and reveal a difference in parametric values. This is where texture analysis software fits in biomedical imaging. 

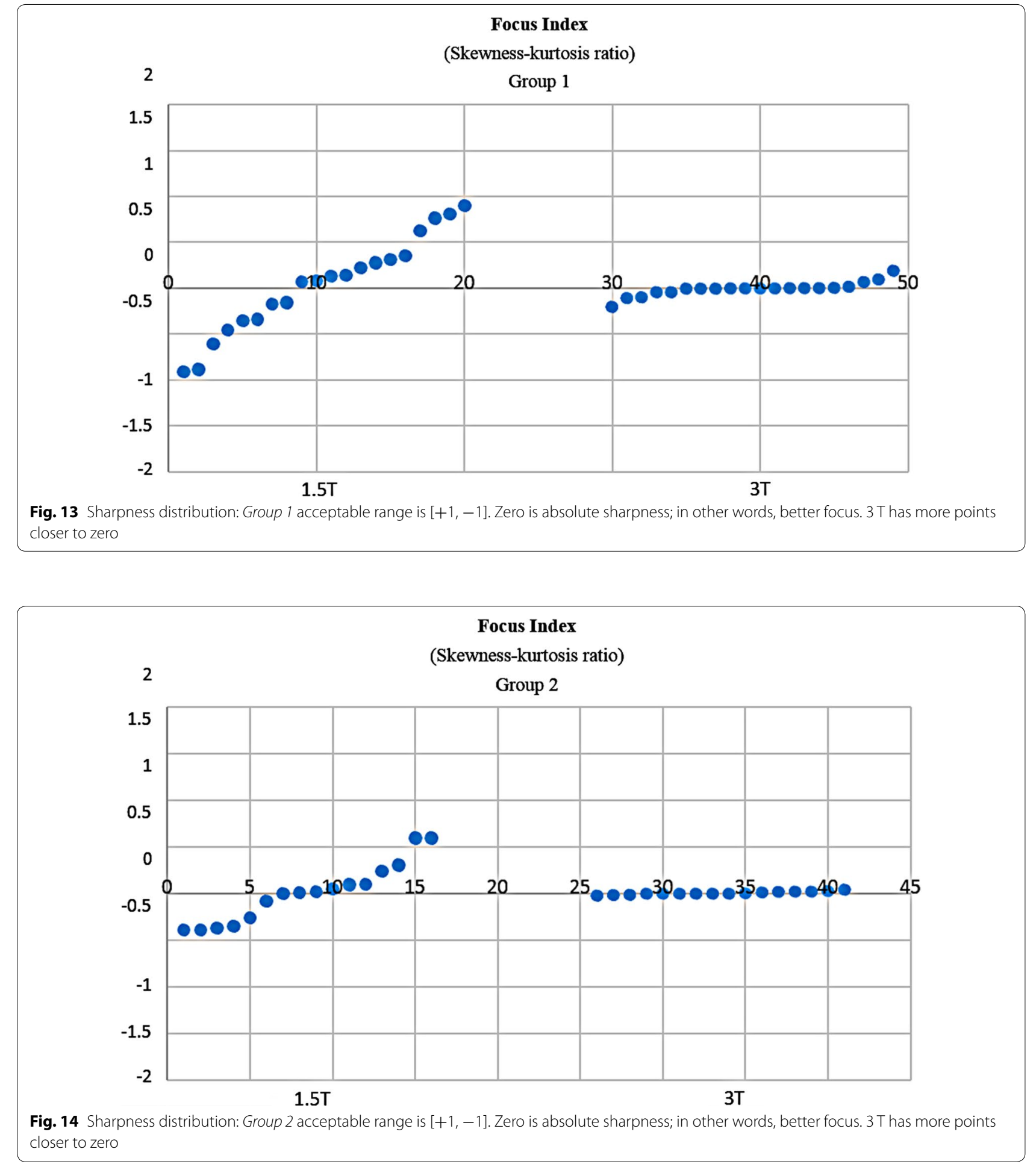

\section{Constraints, limitations, and assumptions}

MaZda Texture Analysis version 5 was used throughout this experiment (Additional file 5). It is a quantitative analysis tool which can extract various parameters from 16-bit DICOM without need of conversion to 8-bit
BMP, and it can measure the actual stored bits within a DICOM container of 16 allocated bits. This feature is crucial in terms of comparing 1.5-3 $\mathrm{T}$ for discriminative power of closely related anatomical structures in fetal brain imaging. Unfortunately, many MaZda parameters 


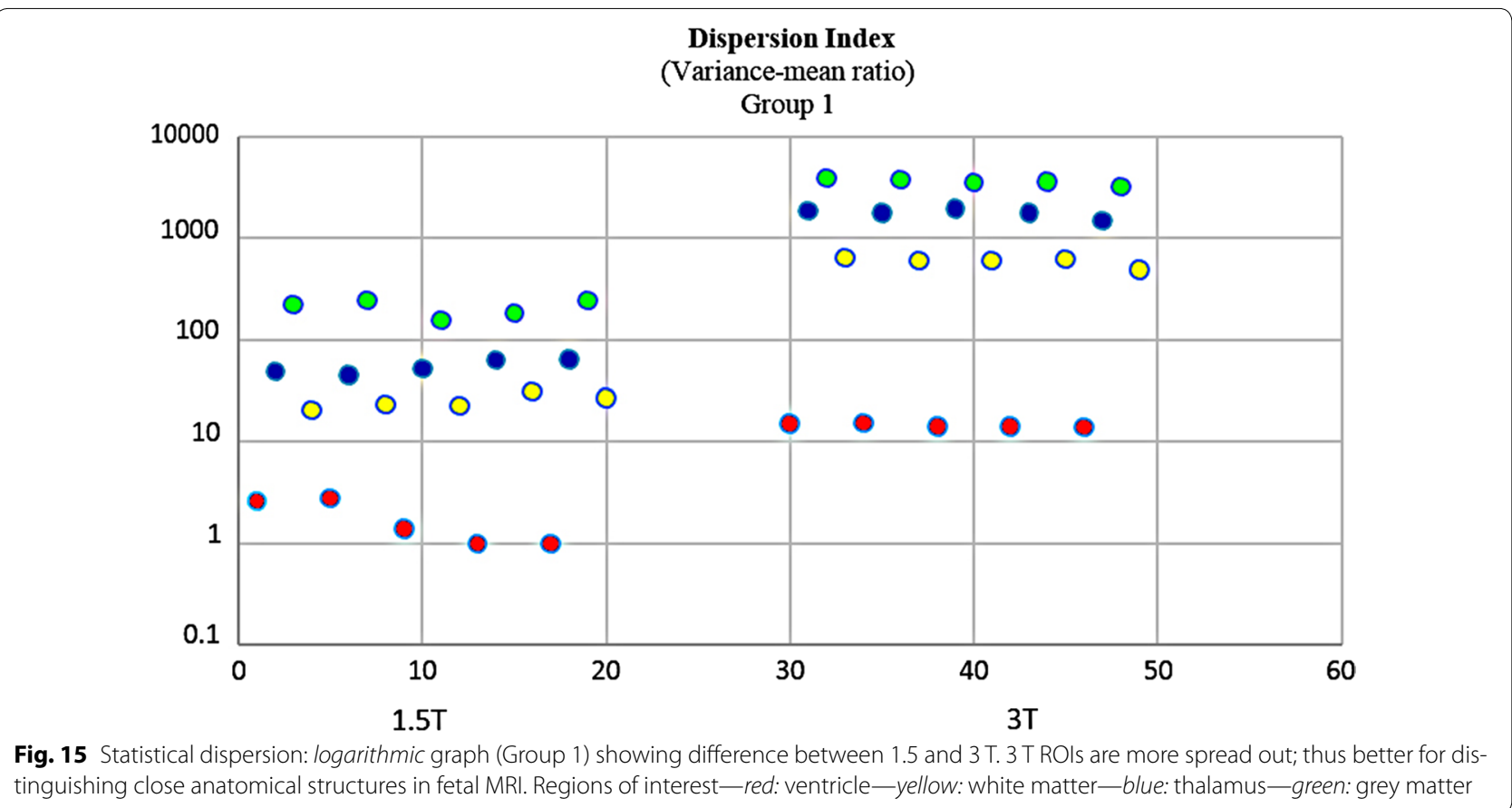

Fig. 15 Statistical dispersion: logarithmic graph (Group 1) showing difference between 1.5 and 3 T. 3 TROls are more spread out; thus better for dis-
tinguishing close anatomical structures in fetal MRI. Regions of interest—red: ventricle—yellow: white matter—blue: thalamus—green: grey matter

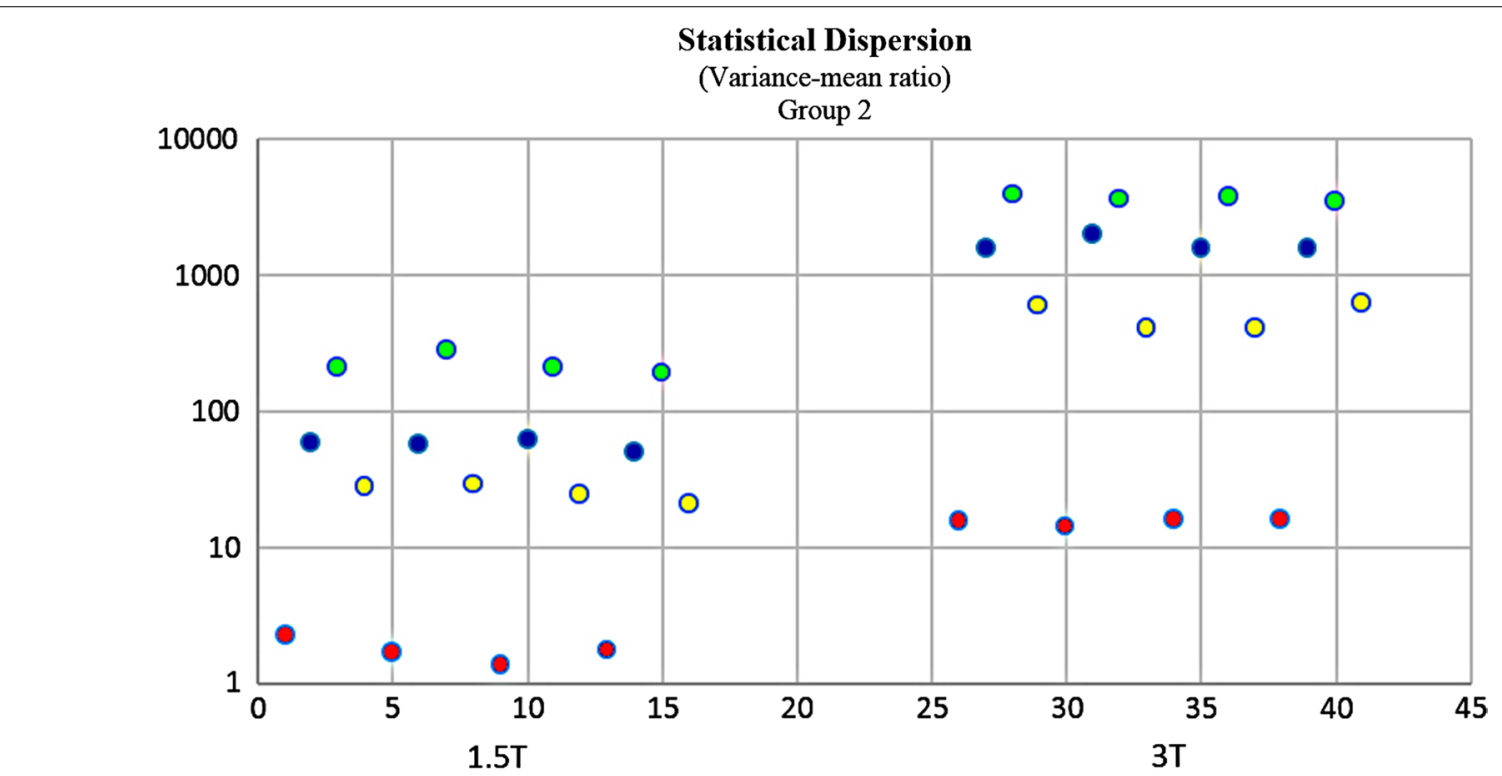

Fig. 16 Statistical dispersion: logarithmic graph (Group 1) showing difference between 1.5 and 3T. 3T ROls are more spread out; thus better for distinguishing close anatomical structures in fetal MRI. Regions of interest—red: ventricle—yellow: white matter—blue: thalamus—green: grey matter

were excluded from this experiment because of either 8-bit or 12-bit limitations. An updated, customized version of this software package would require several manhours and therefore was not feasible in due time.

\section{Recommendations}

As of today, there are still numerous talks about the usefulness of $3 \mathrm{~T}$ MRI in medical practice and the possibility of deleterious effects during early pregnancy. The 
Table 2 The best nine values were selected to determine $p$ values

\begin{tabular}{|c|c|c|c|c|c|c|c|}
\hline \multicolumn{4}{|l|}{$\mathrm{I}_{\text {Focus }}$} & \multicolumn{4}{|l|}{$\mathrm{I}_{\text {DISPERSION }}$} \\
\hline $1.5 \mathrm{~T} \mathrm{G} 1$ & 3 T G1 & 1.5 T G2 & $3 \mathrm{~T} \mathrm{G2}$ & 1.5 T G1 & $3 \mathrm{~T} \mathrm{G1}$ & 1.5 T G2 & 3 T G2 \\
\hline 0.174816 & 0.001664 & 0.261094 & 0.013746 & 245.8439 & 3845.506 & 272.6785 & 3919.487 \\
\hline 0.155916 & 0.001614 & 0.076755 & 0.001496 & 241.6155 & 3725.265 & 208.9656 & 3684.96 \\
\hline 0.07019 & 0.000614 & 0.002159 & 0.000435 & 222.8727 & 3584.081 & 207.59 & 3605.269 \\
\hline 0.084207 & 0.000317 & 0.010039 & $5.16 \mathrm{E}-07$ & 183.2519 & 3518.256 & 191.5476 & 3396.184 \\
\hline 0.12892 & 0.000824 & 0.018455 & $3.76 \mathrm{E}-06$ & 152.8732 & 3234.123 & 62.18858 & 1969.419 \\
\hline 0.14576 & 0.001041 & 0.053909 & 0.00046 & 63.68822 & 1949.152 & 59.11231 & 1557.758 \\
\hline 0.222427 & 0.001126 & 0.095316 & 0.00049 & 62.51141 & 1861.574 & 56.22063 & 1547.73 \\
\hline 0.275593 & 0.002816 & 0.100121 & 0.004151 & 52.44858 & 1775.027 & 50.28433 & 1542.073 \\
\hline 0.315591 & 0.003521 & 0.239347 & 0.01374 & 48.76635 & 1763.935 & 28.30434 & 614.1261 \\
\hline$m=0.175$ & $m=0.0015$ & $m=0.0952$ & $m=0.0038$ & $m=142$ & $m=2806$ & $m=126$ & $m=2426$ \\
\hline $\mathrm{sd}=8 \mathrm{E}-02$ & $\mathrm{sd}=1 \mathrm{E}-03$ & $\mathrm{sd}=9 \mathrm{E}-02$ & $s d=5 E-03$ & $s d=85$ & $s d=935$ & $\mathrm{sd}=92$ & $s d=614$ \\
\hline $\begin{array}{l}t(16)=6.3 \\
p=0.0001\end{array}$ & & $\begin{array}{l}t(16)=2.9 \\
p=0.011\end{array}$ & & $\begin{array}{l}t(16)=-8.5 \\
p=0.0001\end{array}$ & & $\begin{array}{l}t(16)=-5.6 \\
p=0.0001\end{array}$ & \\
\hline
\end{tabular}

scientific views about the differential quality between 1.5 and $3 \mathrm{~T}$ are mixed and perplexed due to subjectivity and unreliable interpretative techniques, which largely depend on the human eye perception. Observational studies have reported that the greater magnetic field of $3 \mathrm{~T}$ produces sharper images based on visual appearance. Such findings are subjected to controversion because different pairs of eyes don't always see the same thing. The normal human eye was reported to have a differential power of a quasi 8-bit scanner. Very few people perceive details beyond that limitation. From a financial point of view, billions of dollars could be saved if $1.5 \mathrm{~T}$ is used instead of 3 T MRI. Nevertheless, financial standpoint and hardship may indeed prevent a clinician from ordering $3 \mathrm{~T}$ MRI, instead of using relevance to beneficiaries as a guide to make such a decision. Some still raise questions over the safety of $3 \mathrm{~T}$ magnetic field in medical practice. Nevertheless, twelve years ago, studies have already shown that $8 \mathrm{~T}$ MRI causes no obvious damage to the human body. Like other imaging modalities, the main issue in the medical community that is affecting the interpretation of $3 \mathrm{~T}$ MR images is subjectivity and observer dependence. It is a challenging task for different human eyes to correctly identify square-pixel variations between the gray-scale image presented in Fig. 1. A key feature of texture analysis with computer vision is that it can quantify texture in pixelated 1.5 and $3 \mathrm{~T}$ MR images into numerical values, which in turn can be used to assess image quality-therefore eliminating subjectivity and reproducibility issues in diagnostic imaging. Some of our guest reviewers had argued that texture quantification was not necessarily an advantage. Yet the World Health Organization (WHO) has noticed a fall in misdiagnosis and treatment cost and a rise in detection and diagnosis of the pandemic disease of tuberculosis (TB) - with introduction of computer-aided interpretation software in regions with prevalent TB outbreak. Again, this research is not about whether human vision is better than artificial vision or vice versa. We disclaim any so-construed allegations. The goal is that texture analysis may assist obstetricians and radiologists in making more accurate and objective medical diagnosis of prenatal pathologies. Reaching this outcome is a matter of testing and finding suitable artificial systems and methods of texture analysis for the appropriate set of images. Last but not least, artificial magnetic fields of $1.5 \mathrm{~T}$ (30,000 times greater than earth's magnetic field) and $3 \mathrm{~T}$ (60,000 times greater than earth's magnetic field) [61], scanners have raised theoretical concerns in the medical community. Unknown risks such as teratogenic and biological effects-if they really exist-could be reduced by using $1.5 \mathrm{~T}$ during the first 28 weeks-as its quality is sufficient [62-67].

\section{Conclusions}

Unquestionably, 3-T exhibits better quality than 1.5-T fetal magnetic resonance imaging. The results were significant $(\mathrm{p}<0.05)$. Nevertheless, $1.5-\mathrm{T}$ is of sufficient quality for routine fetal examination during early pregnancy and therefore can lower the risks of unknown teratogenic effects. Though $3 \mathrm{~T}$ fetal MRI exhibits superior image quality, its usefulness, health claims, side effects, benefits, and safety demand further investigation. 


\section{Additional files}

Additional file 1: Dataset 1. Figure 8 data: stored bits in 1.5/3 T DICOM/
BMP, measured with MaZda version 5 .

Additional file 2: Dataset 2. Effects of pull-down conversion: 12/16-bits grayscale DICOM to 8-bit BMP (RGB-24).

Additional file 3: Dataset 3. Raw texture analysis/Fisher coefficient: > appendix $1, \mathbf{>}$ appendix $2, \mathbf{>}$ appendix 3 .

Additional file 4: Dataset 4. Data for supplemental trial runs: $36 \times 1.5 \mathrm{~T}$; $36 \times 3$ T; group 1: 20-28 weeks; group 2: 29-40 weeks.

Additional file 5. MaZda Package v5 RC HG (release candidate: Hugues Gentillon): this pre-release (beta) version contains: 1) MaZda version 5 for achromatic and chromatic image analysis, 3D editing, feature extraction \& selection, geometrical features for ROls; 2) B11 version 3.3 for feature clustering, image segmentation, unsupervised \& supervised classification.

\section{Abbreviations}

2D: two-dimensional; BMP: bitmap; CNS: central nervous system; ROI: region of interest; CAD: computer-aided diagnosis; COST: cooperation in science and technology; DICOM: digital imaging and communications in medicine; E+: positive exponent; E-: negative exponent; JPEP: Joint Photographic Experts Group; LCD: liquid crystal display; HG: Hugues Gentillon; LS: Ludomir Stefańczyk; MS: Michał Strzelecki; MRL: Maria Respondek-Liberska; MR: magnetic resonance; MRI: magnetic resonance imaging; PD: proton density; RAW: reas as written; RGB: red, blue, green; RGB-24: color space with 8 bits per channel $(8 \times 8 \times 8)$; ROI: region of interest; US: ultrasound; USG: ultrasonography; T: tesla; TB: tuberculosis; $V_{\text {ROI }}$ : variations between ROls; Wk: week.

\section{Authors' contributions}

HG conceived, designed, and carried out the experiment, interpreted the data and drafted the manuscript. Both LS and MRL provided human samples and participated in conceiving/designing the clinical aspects of the research and revising its manuscript. MS provided texture analysis software/plugins and participated in conceiving/designing the technical aspects of the experiment and in revising its manuscript. All authors read and approved the final manuscript.

\section{Author details}

${ }^{1}$ Department of Radiology and Diagnostic Imaging, Barlicki University Hospital, Medical University of Lodz, Lodz, Poland. ${ }^{2}$ Institute of Electronics, The Faculty of Electrical, Electronic, Computer and Control Engineering, Technical University of Lodz, Lodz, Poland. ${ }^{3}$ Diagnosis and Prevention of Congenital Malformations, Instytut Centrum Zdrowia Matki Polki, Lodz, Poland.

\section{Acknowledgements}

The authors gratefully acknowledge prof. Rafał Pawliczak and MUL staff (Division of Doctoral Studies for International Students, Biomedical Sciences, Medical University of Lodz) for research coordination, logistics, and administration; prof. Paweł Liberski and MUL (Division of Doctoral Studies for International Students, Biomedical Sciences, Medical University of Lodz) neuropathology department for counsels with funds to cover MRI expenses; prof. Ludomir Stefańczyk and Barlicki hospital staff (Department of Radiology and Diagnostic Imaging, Barlicki University Hospital, Medical University of Lodz) for sample supply and clinical feedback; prof. Maria Respondek-Liberska and Matki Polki Hospital (Diagnosis and Prevention of Congenital Malformations, Instytut Centrum Zdrowia Matki Polki, Lodz, Poland) for sample supply and clinical feedback - as well as prof. Tadeusz Biegański, ICZMP director; prof. Michał Strzelecki, Sławomir Skonieczka and TUL staff (Institute of Electronics, The Faculty of Electrical, Electronic, Computer and Control Engineering, Technical University of Lodz, Poland) for providing MaZda software and technical feedback.

\section{Competing interests}

The authors declare that there might be unforeseen academic/institutional competing interests-which the authors themselves are not aware of. Nevertheless, this research and its results were carried out independently and thus not endorsed by and in no way influenced by the inventors/patent owner of the MaZda/B11 software and neither financed nor encouraged by COST. Hence it is not intended to be a promotion for COST.

\section{Availability of data and materials}

Links to download raw data are included in this manuscript herein. To get access to medical records, individual solicitor must send formal request to the research committee of the Medical University of Lodz.

Recommended resources: MaZda user manual (http://www.eletel.p.lodz. pl/mazda/download/mazda_manual.pdf); MaZda tutorials (http://www. eletel.p.lodz.pl/programy/mazda/index.php?action=tutorial); MaZda Package v4.6 (http://www.eletel.p.lodz.pl/programy/mazda/); textbook: "Texture analysis for magnetic resonance imaging." 234 pages, ISBN: 80-903660-0-7 Publisher: Med4publishing s.r.o., 2006.

\section{Consent to publish}

Consent for publication was obtained from patients. Furthermore all data from human participants were anonymized as per informed consent agreement. Bioethics Committee Approval Number: RNN/213/13/KE, July 16, 2013.

\section{Ethics approval and consent to participate}

The authors declare that written informed consent was obtained from all patients who were enrolled in the study. Ethics approval was granted by the research committee at the Medical University of Lodz. Agreement number: 3/2011-concluded on December 6, 2011, between the experimenter (Hugues Gentillon), research supervisor (Ludomir Stefańczyk) and rector of Medical University of Lodz (Radzisław Kordek), Faculty of Biomedical Science-Post-graduate research in Diagnostic Imaging and Radiotherapy.

\section{Funding}

Self-funded; material supports from the Medical University of Lodz/Polish Research Committee and affiliated institutions and hospitals; grants and financial aid from Swedish Ministry of Education and Research/Centrala Studiestödsnämnden and from U.S. Department of Education.

Received: 29 February 2016 Accepted: 15 November 2016

Published online: 25 November 2016

\section{References}

1. Hellman LM, Duffus GM, Donald I, Sunden B. Safety of diagnostic ultrasound in obstetrics. Obstet Gynecol Surv. 1971;26(1):13-4.

2. Richards DS. Prenatal ultrasound to detect fetal anomalies. NeoReviews. 2012;13(1):e9-19.

3. Nishi T, Iwasaki M, Yamoto M, Nakano R. Prenatal diagnosis of autosomal recessive polycystic kidney disease by ultrasonography and magnetic resonance imaging. Acta Obstet Gynecol Scand. 1991;70(7-8):615-7.

4. Kawabata I, Takahashi Y, Iwagaki S, Tamaya T. MRI during pregnancy. J Perinat Med. 2003;31(6):449-58.

5. Bulas D, Egloff A. Benefits and risks of MRI in pregnancy. Semin Perinatol. 2013;37(5):301-4.

6. McKenna DA, Meehan CP, Alhajeri AN, Regan MC, O'Keeffe DP. The use of MRI to demonstrate small bowel obstruction during pregnancy. Br J Radiol. 2014;80(949):e11-4.

7. Curtis M, Hopkins MP, Zarlingo T, Martino C, Graceansky-Lengyl M, Jenison EL. Magnetic resonance imaging to avoid laparotomy in pregnancy. Obstet Gynecol. 1993;82:833-6.

8. Quinn TM, Hubbard AM, Adzick NS. Prenatal magnetic resonance imaging enhances fetal diagnosis. J Pediatr Surg. 1998;33(4):553-8.

9. De Wilde JP, Rivers AW, Price DL. A review of the current use of magnetic resonance imaging in pregnancy and safety implications for the fetus. Prog Biophys Mol Biol. 2005;87(2):335-53.

10. Ertl-Wagner B, Lienemann A, Strauss A, Reiser MF. Fetal magnetic resonance imaging: indications, technique, anatomical considerations and a review of fetal abnormalities. Eur Radiol. 2002;12(8):1931-40.

11. McCarthy SM, Filly RA, Stark DD, Hricak H, Brant-Zawadzki MN, Callen PW, Higgins CB. Obstetrical magnetic resonance imaging: fetal anatomy. Radiology. 1985;154(2):427-32. 
12. Weinreb JC, Lowe TW, Santos-Ramos R, Cunningham FG, Parkey R. Magnetic resonance imaging in obstetric diagnosis. Radiology. 1985;154(1):157-61

13. Huisman TA, Martin E, Kubik-Huch R, Marincek B. Fetal magnetic resonance imaging of the brain: technical considerations and normal brain development. Eur Radiol. 2002;12(8):1941-51.

14. Hartwig V, Giovannetti G, Vanello N, Lombardi M, Landini L, Simi S. Biological effects and safety in magnetic resonance imaging: a review. Int J Environ Res Public Health. 2009;6(6):1778-98.

15. Kok RD, de Vries MM, Heerschap A, van den Berg PP. Absence of harmfu effects of magnetic resonance exposure at $1.5 \mathrm{~T}$ in utero during the third trimester of pregnancy: a follow-up study. Magn Reson Imaging. 2004;22(6):851-4.

16. Baker LC, Wheeler SK. Managed care and technology diffusion: the case of MRI. Health Aff. 1998;17(5):195-207.

17. Abe H, Schmidt RA, Shah RN, Shimauchi A, Kulkarni K, Sennett CA, Newstead GM. MR-directed ("Second-Look") ultrasound examination for breast lesions detected initially on MRI: MR and sonographic findings. Am J Roentgenol. 2010;194(2):370-7.

18. Carmichael IW, MacLeod AM, Travlos J. MRI can prevent unnecessary arthroscopy. J Bone Joint Surg Br. 1997;79(4):624-5.

19. Lubovsky O, Liebergall M, Mattan Y, Weil Y, Mosheiff R. Early diagnosis of occult hip fractures: MRI versus CT scan. Injury. 2005;36(6):788-92.

20. Naredo E, Collado P, Cruz A, Palop MJ, Cabero F, Richi P, et al. Longitudinal power Doppler ultrasonographic assessment of joint inflammatory activity in early rheumatoid arthritis: predictive value in disease activity and radiologic progression. Arthritis Care Res. 2007:57(1):116-24.

21. Battista R, Banta HD, Jonsson E, Hodge M, Gelband H. Lessons from the eight countries. In: Health care technology and its assessment in eight countries, OTA-BP-H-140, chap 7. US Congress, Office of Technology Assessment. Washington, DC: US Government Printing Office, DIANE Publishing. 1995; p. 209-58.

22. Girouard N, Imai Y. The health care system in Poland. OECD, Economics Department Working Papers. 2000. p. 257. doi: 10.1787/265022143317.

23. Lewis MA. Who is paying for health care in Eastern Europe and Central Asia?, vol. 20940. Washington: World Bank Publications; 2000. p. 1-31.

24. Materka A, Strzelecki M. Texture analysis methods-a review, Technical University of Lodz, Institute of Electronics. COST, Brussels. 1998;B11 report:1998.

25. Szczypinski P, Strzelecki M, Materka A, Klepaczko A. MaZda - A software package for image texture analysis. Comput Methods Programs Biomed. 2009;94(1):66-76.

26. Strzelecki M, Szczypinski P, Materka A, Klepaczko A. A software tool for automatic classification and segmentation of 2D/3D medical images. Nucl Instrum Methods Phys Res Sect A. 2013;702:137-40.

27. Muyoyeta M, Maduskar P, Moyo M, Kasese N, Milimo D, Spooner R, et al. The sensitivity and specificity of using a computer aided diagnosis program for automatically scoring chest $X$-rays of presumptive TB patients compared with Xpert MTB/RIF in Lusaka, Zambia. PLoS ONE. 2014;9(4):e93757.

28. Maduskar P, Muyoyeta M, Ayles H, Hogeweg L, Peters-Bax L, van Ginneken B. Detection of tuberculosis using digital chest radiography: automated reading vs. interpretation by clinical officers. Int J Tuberc Lung Dis. 2013;17(12):1613-20.

29. Breuninger M, van Ginneken B, Philipsen RH, Mhimbira F, Hella JJ, Lwilla F, et al. Diagnostic accuracy of computer-aided detection of pulmonary tuberculosis in chest radiographs: a validation study from sub-Saharan Africa. PLoS ONE. 2014;9(9):e106381.

30. Hillemann D, Rüsch-Gerdes S, Boehme C, Richter E. Rapid molecular detection of extrapulmonary tuberculosis by the automated GeneXpert MTB/RIF system. J Clin Microbiol. 2011;49(4):1202-5.

31. Steiner A, Mangu C, van den Hombergh J, van Deutekom $H$, van Ginneken $B$, Clowes P, Hoelscher M. Screening for pulmonary tuberculosis in a Tanzanian prison and computer-aided interpretation of chest $X$-rays. Public Health Action. 2015;5(4):249.

32. Philipsen RHHM, Sánchez Cl, Maduskar P, Melendez J, Peters-Bax L, Peter JG, van Ginneken B. Automated chest-radiography as a triage for Xpert testing in resource-constrained settings: a prospective study of diagnostic accuracy and costs. NPG Sci Rep. 2015;5:12215. doi:10.1038/ srep 12215
33. Lawn SD, Mwaba P, Bates M, Piatek A, Alexander H, Marais BJ, Abubakar I. Advances in tuberculosis diagnostics: the Xpert MTB/RIF assay and future prospects for a point-of-care test. Lancet Infect Dis. 2013;13(4):349-61.

34. Blehm C, Vishnu S, Khattak A, Mitra S, Yee RW. Computer vision syndrome: a review. Surv Ophthalmol. 2005;50(3):253-62.

35. Livingstone $\mathrm{M}, \mathrm{Hubel} \mathrm{DH}$. Vision and art: the biology of seeing ( $\mathrm{vol} 2$ ), chapter 12. New York: Harry N. Abrams; 2002

36. Watson AB, Null CH (1997) Digital images and human vision. Nasa Res Center. 1997;20020041928:El'97.

37. Graps A. An introduction to wavelets. IEEE Comput Sci Eng. 1995;2(2):50-61.

38. Akay M. Wavelet applications in medicine. IEEE Spectr. 1997;34(5):50-6.

39. Bharati MH, Liu JJ, MacGregor JF. Image texture analysis: methods and comparisons. Chemometr Intell Lab Syst. 2004;72(1):57-71.

40. Castellano G, Bonilha L, Li LM, Cendes F. Texture analysis of medical images. Clin Radiol. 2004;59(12):1061-9.

41. Hendrick RE. Artifacts and errors in breast magnetic resonance imaging. Breast MRI: fundamentals and technical aspects, ch 11. 2008. p. 187-207.

42. Herbst M, Maclaren J, Lovell-Smith C, Sostheim R, Egger K, Harloff A, et al. Reproduction of motion artifacts for performance analysis of prospective motion correction in MRI. Magn Reson Med. 2014;71(1):182-90.

43. Somasundaram K, Kalavathi P. Analysis of imaging artifacts in MR brain images. Oriental J Comput Sci Technol. 2012;5(1):135-41.

44. Zhuo J, Gullapalli RP. MR artifacts, safety, and quality control. Radiographics. 2006:26(1):275-97.

45. Kohlenberg L. Everybody knows this is no wear. Am Journalism Rev. 1994;16(4):38-42

46. Cromey DW. Avoiding twisted pixels: ethical guidelines for the appropriate use and manipulation of scientific digital images. Sci Eng Ethics. 2010;16(4):639-67.

47. Shotton J, Johnson M, Cipolla R. Semantic texton forests for image categorization and segmentation. In: IEEE conference on computer vision and pattern recognition, CVPR 2008; 2008. p. 1-8.

48. Pal NR, Pal SK. A review on image segmentation techniques. Pattern Recogn. 1993;26(9):1277-94.

49. Zakrzewski M. Polish doctors implanted the world's smallest artificial cardiac pacemaker. University Hospital of Poznan, EPA. 2016; Press Release. p. 52543790 .

50. Majda T. Polish cardiology started in Lodz 60 years ago-1950-2010. Cardiol J. 2011;18(1):110-3.

51. Hrynaszkiewicz I, Norton ML, Vickers AJ, Altman DG. Preparing raw clinical data for publication: guidance for journal editors, authors, and peer reviewers. BMJ. 2010;340(8):c181.

52. Rose A. Are face-detection cameras racist? Time magazine online. (http:// www.time.com/time/business/article/0,8599,1954643-1,00.html). 22 Jan 2010;1954643-1.

53. Whitehill J, Bartlett MS, Movellan JR. Automatic facial expression recognition. Social Emot Nat Artifact. 2013;ch6:88-9.

54. Frayer M. Giving the toaster eyes: applied anthropomorphism and its influences on user-object relations with everyday objects. 2010; KOVM02 20101:id1604671.

55. McKenna DA, Meehan CP, Alhajeri AN, Regan MC, O'Keeffe DP. The use of MRI to demonstrate small bowel obstruction during pregnancy. $\mathrm{Br} J$ Radiol. 2007;80(949):e11-4.

56. Katsura S, Mizokami Y, Yaguchi H. Perceived quality of wood images influenced by the ratio of skewness to kurtosis of image histogram. The 1st Asia color association conference, 2013, Proc. p. 86-9.

57. Katsura S, Mizokami Y, Yaguchi H. Perceived quality of wood images influenced by the skewness of image histogram. Opt Rev. 2015:22(4):565-76.

58. Law WK, Chung AC. Minimal weighted local variance as edge detector for active contour models. In: Computer vision-ACCV, 2006; Berlin: Springer; 2006. p. 622-632.

59. Schmitz BL, Aschoff AJ, Hoffmann MH, Grön G. Advantages and pitfalls in 3 T MR brain imaging: a pictorial review. Am J Neuroradiol. 2005:26(9):2229-37.

60. Materka A. Texture analysis methodologies for magnetic resonance imaging. Dialogues Clin Neurosci. 2004;6(2):243-50 
61. Jerrolds J, Keene S. MRI safety at 3 versus 1.5 T. Internet J World Health Soc Politics. 2009;6:1.

62. Cannie M, Jani J, Dymarkowski S, Deprest J. Fetal magnetic resonance imaging: luxury or necessity? Ultrasound Obstet Gynecol. 2006;27(5):471-6.

63. Levine D, Barnes PD, Robertson RR, Wong G, Mehta TS. Fast MR imaging of fetal central nervous system abnormalities 1. Radiology. 2003;229(1):51-61.

64. Griffiths PD, Paley MN, Whitby EH. MR imaging of the fetal brain and spine: a maturing technology. Ann Acad Med Singapore. 2003;32(4):483-9.
65. Whitby EH, Paley MNJ, Sprigg A, Rutter S, Davies NP, Wilkinson ID, Griffiths PD. Comparison of ultrasound and magnetic resonance imaging in 100 singleton pregnancies with suspected brain abnormalities. BJOG Int J Obs Gynaecol. 2004;111(8):784-92.

66. Altman RP, Randolph JG, Lilly JR. Sacrococcygeal teratoma: American Academy of Pediatrics surgical section survey-1973. J Pediatr Surg. 1974;9(3):389-98.

67. Avni FE, Guibaud L, Robert Y, Segers V, Ziereisen F, Delaet MH, Metens T. MR imaging of fetal sacrococcygeal teratoma: diagnosis and assessment. Am J Roentgenol. 2002;178(1):179-83.

\section{Submit your next manuscript to BioMed Central and we will help you at every step:}

- We accept pre-submission inquiries

- Our selector tool helps you to find the most relevant journal

- We provide round the clock customer support

- Convenient online submission

- Thorough peer review

- Inclusion in PubMed and all major indexing services

- Maximum visibility for your research

Submit your manuscript at www.biomedcentral.com/submit 